\title{
Stability Analysis for a Type of Multiswitching System with Parallel Structure
}

\author{
Zhang Yan $\mathbb{D}^{1,2}$ Liu Yongqiang $\mathbb{D}^{1},{ }^{1}$ and Liu Yang $\mathbb{D}^{1}$ \\ ${ }^{1}$ School of Electric Power, South China University of Technology, Guangzhou, Guangdong, China \\ ${ }^{2}$ Centre of Faculty Development and Educational Technology, Guangdong University of Finance and Economics, \\ Guangzhou, Guangdong, China
}

Correspondence should be addressed to Liu Yongqiang; epyqliu@scut.edu.cn

Received 28 March 2018; Revised 28 June 2018; Accepted 25 July 2018; Published 8 August 2018

Academic Editor: Asier Ibeas

Copyright (C) 2018 Zhang Yan et al. This is an open access article distributed under the Creative Commons Attribution License, which permits unrestricted use, distribution, and reproduction in any medium, provided the original work is properly cited.

\begin{abstract}
This paper proposes a new type of multiswitching system and a subsystems-group as a basic switching unit that obeys the law. Unlike traditional switching systems, the system selects multiple subsystems instead of one on each time interval. Thus, a framework of parallel structure organizes the subsystems as a group. A multiswitched system is widely used in engineering for modelling and control; this system reflects the actual industrial dynamical process. Thus, the stability of the system is studied. Assuming that these continuous and discrete-time subsystems are Hurwitz and Schur stable, the subsystems-groups matrices commute each other based on the subsystems matrices pairwise commutative. Then, the multiswitched system is exponentially stable under arbitrary switching, and there exists a common Lyapunov function for these subsystems. The main result is extended to a parallel-like structure; therefore, some stability results are gained under some reasonable assumption. At last, a numeral example is given to illustrate the structure and the stability of this system.
\end{abstract}

\section{Introduction}

A switched system is a particular kind of hybrid dynamical system that consist of a finite number of subsystems and a switching signal that switches between these subsystems in an orderly manner. The set of subsystems can be continuoustime and discrete-time; the mathematical models are differential equations and difference equations. According to the dynamics behaviours, it can be classified as continuous-time switched system, discrete-time switched system, or mixed switched system (both them) [1], linear or nonlinear and so on [2]. Actually, many fields of complex industrial processes include typical switching systems, which reflect the hybrid dynamical characteristics that are widely used in many fields [3-8], such as in aircraft control, the automotive industry, communication systems, electrical engineering, chemical processes, and so on.

Multiswitched systems are a novel type of system, which can be composed of a set of continuous-time subsystemsgroups

$$
\dot{x}(t)=E_{c i} x(t), \quad c i=c 1, \ldots, c N_{c}
$$

$$
E_{c i}=\left(\begin{array}{ccccc}
A_{c 1} & \cdots & 0 & \cdots & 0 \\
\vdots & \ddots & & & \vdots \\
0 & & A_{c p} & & 0 \\
\vdots & & & \ddots & \vdots \\
0 & \cdots & 0 & \cdots & A_{c M}
\end{array}\right),
$$

$$
c p=c 1, \ldots, c M
$$

and a set of discrete-time subsystems-groups

$$
\begin{gathered}
x(k+1)=E_{d j} x(k), \quad d j=d 1, \ldots, d N_{d} \\
E_{d j}=\left(\begin{array}{ccccc}
A_{d 1} & \cdots & 0 & \cdots & 0 \\
\vdots & \ddots & & & \vdots \\
0 & & A_{d q} & & 0 \\
\vdots & & & \ddots & \vdots \\
0 & \cdots & 0 & \cdots & A_{d M}
\end{array}\right),
\end{gathered}
$$


where $x(t), x(k)$ are the states, $t$ is the time scalar, and $\tau$ is positive integer of the sample period. $E_{c i}$ and $E_{d j}$ are nonnull constant matrices with appropriate dimensions, which are combined by some constant matrices $A_{c p}$ and $A_{d q}$, respectively. Subsystems-groups (1) and (2) are composed of continuoustime subsystems $\dot{x}(t)=A_{c p} x(t)$ with $c p=c 1, \ldots, c M$ and discrete-time subsystems $x(k+1)=A_{d q} x(k)$ with $d q=d 1, \ldots, d M$, respectively. The system switches between matrices $E_{c 1}, E_{c 2}, \ldots, E_{c N_{c}}, E_{d 1}, E_{d 2}, \ldots, E_{d N_{d}}$ belonging to the set $E ; E \triangleq\left\{E_{c}, E_{d}\right\}, E_{c} \triangleq\left\{E_{c 1}, E_{c 2}, \ldots, E_{c N_{c}}\right\}$, and $E_{d} \triangleq\left\{E_{d 1}, E_{d 2}, \ldots, E_{d N_{d}}\right\} . A_{c 1}, A_{c 2}, \ldots, A_{c M}$ belong to the set $A_{c} \triangleq\left\{A_{c 1}, A_{c 2}, \ldots, A_{c M}\right\}, A_{d 1}, A_{d 2}, \ldots, A_{d M}$ belong to the set $A_{d} \triangleq\left\{A_{d 1}, A_{d 2}, \ldots, A_{d M}\right\}$, and $A \triangleq\left\{A_{d}, A_{c}\right\}$. $A$ and $E$ are finite sets.

The major difference between a multiswitched system and a traditional switched system is that the former selects any amount of subsystems, and the latter selects only one each time. There is a structure for organizing the subsystems as a subsystems-group on each interval that includes mutual independence between subsystems. Many different structures can be considered, such as parallel, tandem, and other mixed modes. Equations (2) and (4) show the parallel structure of the matrices. However, the traditional switching system has only one matrix without any structure.

A multiswitched system is widely used in engineering cases, such as in the chilled water system in central air conditioning. The dynamical pumping process is a kind of typical multiswitch system, and each different pump as a subsystem with switching and variable frequency behaviours. For variable flow control, there are several different pumps working together as a subsystem-group at each time period. According to the water pipe network, the pumps are parallel in structure. Thus, this physical model illustrates the proposed system. In this paper, only parallel and parallel-like structures are researched.

Modelling [9], performance analysis [10] and optimal control [11, 12] are the key issues of switched systems. Obviously, stability analysis is a very important research branch that has attracted the attention of researchers globally. Stability is the most basic property and the primary issue to be solved by the control system. The stability problems of switched systems are reduced to the following three basic issues [13]: finding conditions to guarantee asymptotical stability for any switching signal; identifying some switching signals for asymptotical stability; and constructing a switching signal to make the system asymptotically stable. Accordingly, some excellent theoretical methods are proposed for solving those problems, such as common Lyapunov functions $[14,15]$, multiple Lyapunov functions [16-18], dwell time and average dwell time $[19,20]$, and piecewise quadratic Lyapunov functions [16].

It is worth noting that stability under arbitrary switching is a fundamental in the design and analysis of switched systems [21]. It is known that all the subsystems that are exponentially stable are not sufficient to guarantee stability under arbitrary switching, expect for some reasonable assumptions, such as the state matrices of subsystems commute pairwise $[2,14]$ (e.g., $A_{i} A_{j}=A_{j} A_{i}$, for all $i, j \in I, i \neq j ; A_{c i} A_{d j}=$ $A_{d j} A_{c i}$, for all $i, j \in I, \mathrm{c}$ and d denote continuous-time and discrete-time subsystems, respectively); the state matrices of subsystems are symmetric [22] (e.g., $A_{i}=A_{i}{ }^{T}$, for all $i \in I$ ); the state matrices of subsystems are normal [23] (e.g., $A_{c i}{ }^{T} A_{c i}=A_{c i} A_{c i}{ }^{T}$, for all $i \in I$ and $A_{d j}{ }^{T} A_{d j}=$ $A_{d j} A_{d j}{ }^{T}$, for all $j \in I, c$ and $d$ denote the same substances above). Thus, some researchers present a sufficient condition in terms of the Lie algebra. If this Lie algebra is solvable, the exponential stability of the switched system for arbitrary switching can be gained [20]. A more general result is that the semisimple subalgebra $S$ is a compact Lie algebra by considered Levi decomposition, and the exponentially stability can be ensured [24]. Previous research shows that the pairwise commutation of the vector fields is a sufficient condition for the stability [25]. By using Hurwitz stable matrix pencils $\sigma_{\alpha}\left[A_{1}, A_{2}\right]$ and $\sigma_{\alpha}\left[A_{1}, A_{2}{ }^{-1}\right]$, a common quadratic Lyapunov function exists that can guarantee stability under arbitrary switching [26]. Based on previous research, the main emphasis is placed on the conditions of stability analysis and a common Lyapunov function is constructed under an assumption.

In this paper, a type of linear multiswitched system with parallel structure is put forward for the first time. The composition of the system is expounded, and the structure and form of the subsystems-group are described. From the switching law and the physical meaning, we illustrate the difference between the system and the traditional switching system. A case of chilled water system pumps in central air conditioning shows the engineering significance of the system. Next, stability under arbitrary switching for this type of system is studied. The inference of properties of state matrices of the subsystems-group is derived from the subsystems. Then, based on the conditions of Hurwitz stable and Schur stable for continuous-time subsystems and discretetime subsystems, respectively, the stability of subsystemsgroups can be obtained. The matrices $E_{c}$ and $E_{d}$ of the subsystems-groups commute pairwise can be ensured based on the assumptions of the state matrices $A_{c}$ and $A_{d}[2,14]$. Thus, the stability under arbitrary switching is guaranteed, and a common Lyapunov function is given for all subsystemsgroups and subsystems. Finally, the result is extended to a parallel-like structure, which reduces conservativeness in stability analysis.

The body of this paper is organized as follows: in Section 2, the system description, the example of the multiswitching system, and the preliminaries are presented. In Section 3, the main stability results in the continuous-time system and the mixed system are expressed; two examples are also given to illustrate the results. In Section 4, the studies extended to parallel-like structure is presented. Section 5 concludes the paper.

\section{System Description and Mathematical Preliminaries}

2.1. System Description and Examples. Throughout, the following notation is adopted: $\mathbb{R}$ and $\mathbb{C}$ denote the fields of real and complex number, respectively; $\mathbb{R}^{n}$ denotes the $n$ dimensional real Euclidean space; $\mathbb{R}^{n \times n}$ denotes the space of 
$n \times n$ matrices with real entries; $x_{i}$ and $x_{j}$ denote the $i^{\text {th }}$ and $j^{\text {th }}$ components of the vector $x$ in $\mathbb{R}^{n}$, respectively; $a_{c p p^{\prime}}$ and $a_{d q q^{\prime}}$ denote the entry in the $\left(p, p^{\prime}\right)$ and $\left(q, q^{\prime}\right)$ position of the matrices $A$ or $E$ in $\mathbb{R}^{n \times n}$, respectively.

There are three examples to show the multiswitched system and the parallel structure.

Example 1. Consider a set $A=\left\{A_{1}, A_{2}, A_{3}\right\}$ of a switched system, which are the constant matrices of 3 subsystems, respectively. Assume in the classical switched system that there are 3 subsystems. The system switches between $A_{1}, A_{2}$, and $A_{3}$. Assume in the multiswitched system that there are 7 subsystems-groups. The system switches between $E$,

$$
\begin{aligned}
& E=\left\{E_{1}, E_{2}, E_{3}, E_{4}, E_{5}, E_{6}, E_{7}\right\}=\left\{\left[\begin{array}{ccc}
A_{1} & 0 & 0 \\
0 & 0 & 0 \\
0 & 0 & 0
\end{array}\right],\right. \\
& {\left[\begin{array}{lll}
0 & 0 & 0 \\
0 & A_{2} & 0 \\
0 & 0 & 0
\end{array}\right],\left[\begin{array}{lll}
0 & 0 & 0 \\
0 & 0 & 0 \\
0 & 0 & A_{3}
\end{array}\right],\left[\begin{array}{ccc}
A_{1} & 0 & 0 \\
0 & A_{2} & 0 \\
0 & 0 & 0
\end{array}\right],} \\
& \left.\left[\begin{array}{ccc}
A_{1} & 0 & 0 \\
0 & 0 & 0 \\
0 & 0 & A_{3}
\end{array}\right],\left[\begin{array}{ccc}
0 & 0 & 0 \\
0 & A_{2} & 0 \\
0 & 0 & A_{3}
\end{array}\right],\left[\begin{array}{ccc}
A_{1} & 0 & 0 \\
0 & A_{2} & 0 \\
0 & 0 & A_{3}
\end{array}\right]\right\} \\
& \text { Here, } N_{c}=C_{c M}^{1}+\cdots+C_{c M}^{c M} \text { and } N_{d}=C_{d M}^{1}+\cdots+C_{d M}^{d M} .
\end{aligned}
$$

Remark 2. A continuous-time subsystems-group composed of a subsystem or all the subsystems. Obviously, there are subsystems, but the value is null. It is same with a discretetime subsystems-group.

Remark 3. Continuous-time subsystems cannot mix with discrete-time subsystems to be a subsystems-group. Continuous-time subsystems-groups and discrete-time subsystemsgroups must be distinguished.

Remark 4. When $E_{c i}$ is a singular matrix, zero rows or zero columns remain with the aim of uniform description. In Example 1, $E_{1}=A_{1}$, but the uniform description is kept to show $E_{1}=\left[\begin{array}{ccc}A_{1} & 0 & 0 \\ 0 & A_{2} & 0 \\ 0 & 0 & A_{3}\end{array}\right]=\left[\begin{array}{ccc}A_{1} & 0 & 0 \\ 0 & 0 & 0 \\ 0 & 0 & 0\end{array}\right]$, with $A_{2}=0, A_{3}=0$. Thus, all $E_{c i}$ values look the same, $c i=c 1, \ldots, c N_{c}$. The same is true of $E_{d j}$.

Remark 5. Equations (2) and (4) are standard parallel structures. A parallel-like structure will be considered in Section 4, which has a coupling phenomenon based on a parallel structure between some subsystems. Assumptions are made for reducing conservativeness to obtain ideal stability.

Example 6. In a chilled water system of a central air conditioning system, there are three pumps driving the chilled water from the evaporator to the air conditioning unit (see Figure 1). All the pumps can be switched ON/OFF and

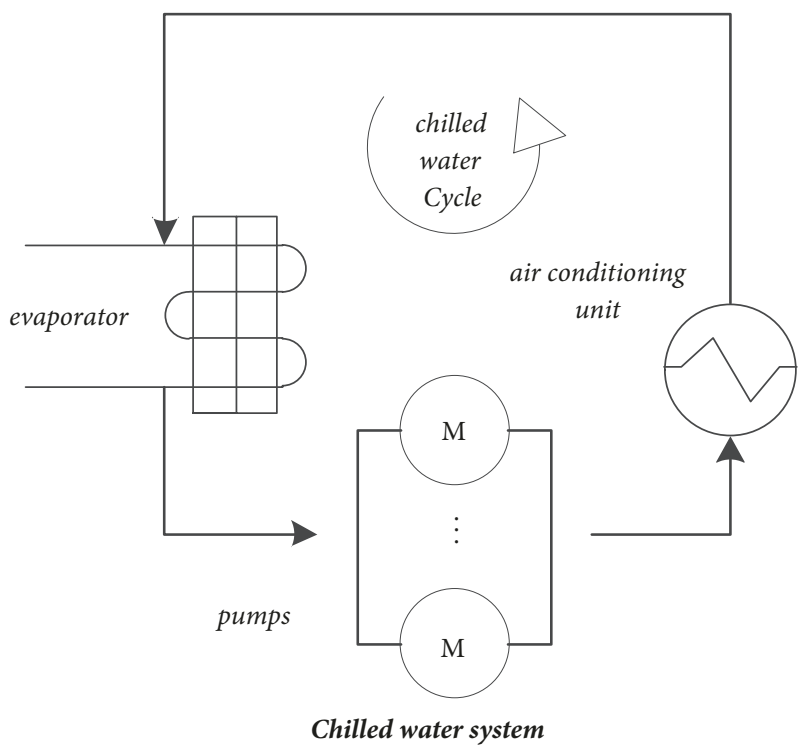

FIGURE 1: Chilled water system of central air conditioning.

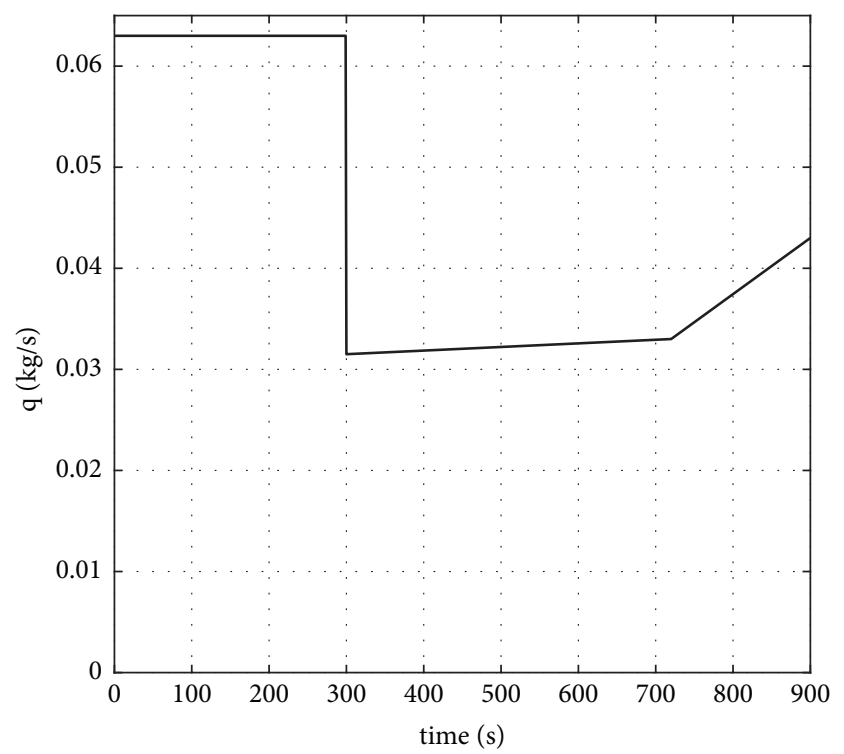

FIGURE 2: Total flow volume.

the variable frequency obeying a range of 50\%-100\% rated frequency. For setting an energy-saving control strategy with variable water volume technology, the whole volume cannot be less than $50 \%$ of the rated volume. Assuming in a time interval that the cold load changes, the switching, and pump water volume are shown in Figures 2 and 3. The parameters and symbols are shown in Table 2.

It is assumed that the cold load in a certain area requires air conditioning to meet the cold demand within 5 minutes to achieve a balanced state. Then, in the first 5 minutes, 3 pumps work at the rated frequency. After reaching the preset value, the cold load is stable with small fluctuations. In this period, pump $A$ and $B$ exit; only pump $C$ works in the form of frequency conversion, maintaining the driving chilled water and transferring cooling capacity. In the last 3 minutes, due 


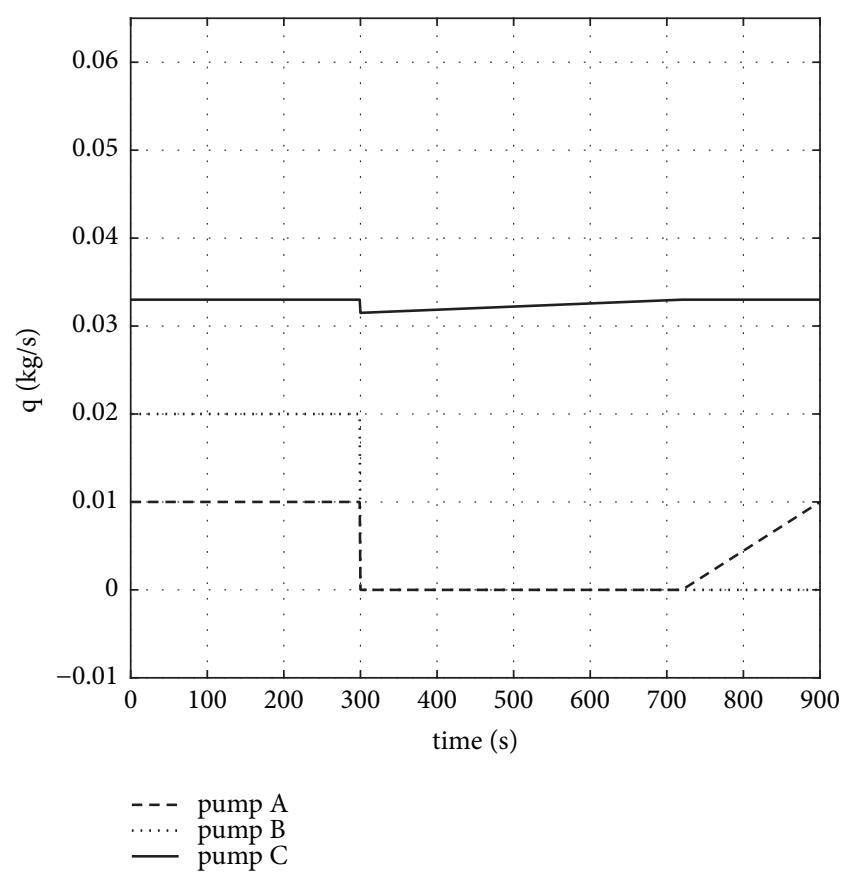

FIgURE 3: Water volumes of pumps.

to the cold load increase, a pump is not enough to meet the demand, and the system puts pump $A$ into work with pump $C$ together with frequency conversion.

From the three time intervals ([0,5 $\mathrm{min}]$, [5 min, $12 \mathrm{~min}]$, and [ $12 \mathrm{~min}, 15 \mathrm{~min}])$, we find that there are three, one, and two subsystems working, respectively. According to the framework in this paper, the three pumps denote different continuous-time subsystems, and the work combinations of the pumps denote the different continuous-time subsystemsgroups. Assuming the pump $A, B$, and $C$ denote the subsystem $X_{1}, X_{2}$, and $X_{3}$, respectively; and the combination $A / B / C$, $C$ and $A / C$ we just said denote the subsystems-group $Y_{1}, Y_{2}$, and $Y_{3}$, respectively.

Example 7. In the same system with Example 6, but the pumps are divided into one variable frequency pump and two switchable fixed frequency pumps. Thus, the former is a continuous-time subsystem, and the latter are discrete-time subsystems. According to the energy-saving control strategy, assuming pump $C$ is always working with variable frequency, and pumps $A$ and $B$ are switched ON or OFF with fixed frequency. Thus, the chilled water system is composed of continuous and discrete-time subsystems. The parameters and symbols are shown in Table 3.

There are two simulation figures (Figures 4 and 5) that illustrate the switching and working situations of the system.

In the first 5 minutes, 3 pumps work together; this seems a subsystems-group with one continuous and two discrete-time subsystems. In the interval of from $5 \mathrm{~min}$ to $12 \mathrm{~min}$, only pump $C$ works, which is the only one continuous-time subsystem that is a subsystems-group. In the last three minutes, pump $A$ works with pump $C$; therefore, the

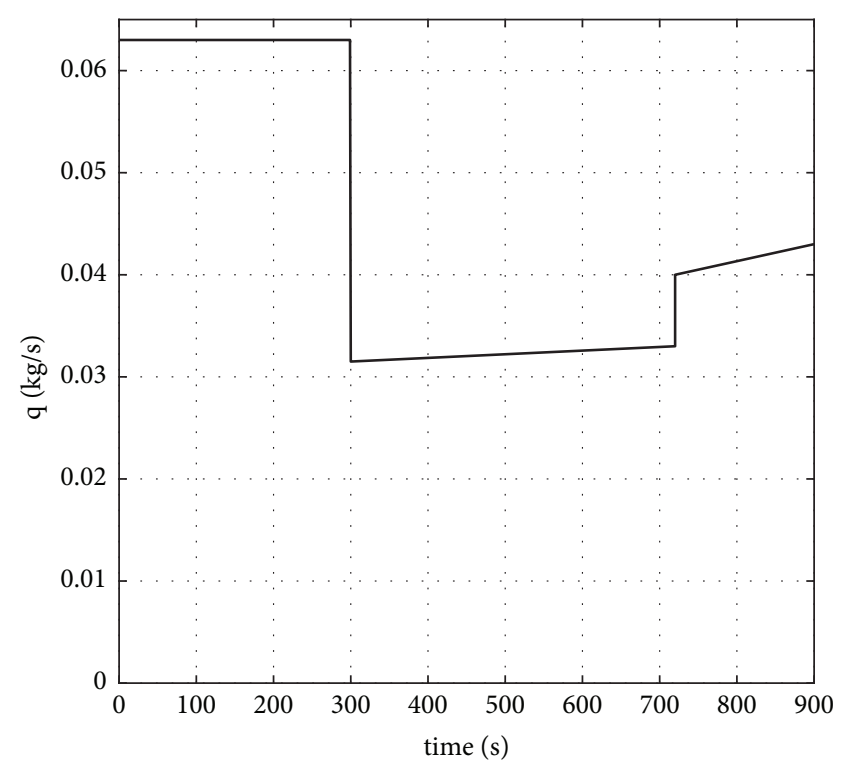

FIGURE 4: Total flow volume.

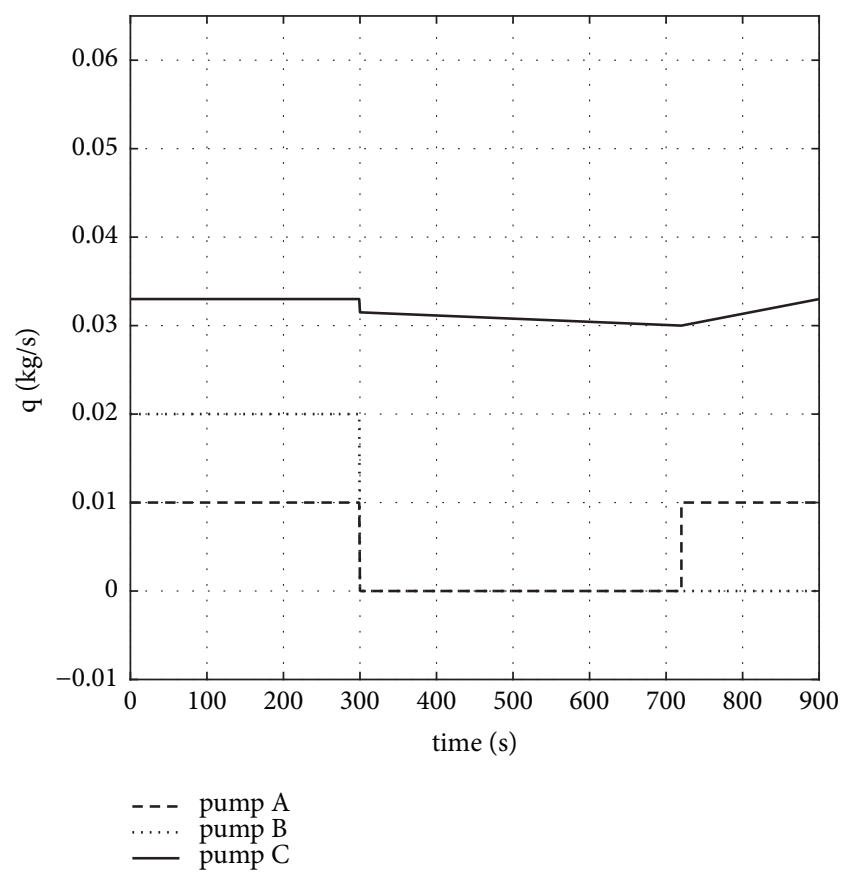

FIgURE 5: Pump water volume.

subsystems-group has one continuous and one discrete-time subsystem.

2.2. Important Theories for Stability Analysis. We analyse the stability of the multiswitched system based on the structural property of E-matrices, which cannot exist without the basic component, $A$-matrices. All the inferences of multiswitched system are derived from a classical switched system. Thus, some important theorems and propositions of the classical switched system should be quoted first. 
Theorem 8 (see [14]). Consider a classical continuous-time switched system $\dot{x}=A_{c} x(t)$ with $A_{c} \triangleq\left\{A_{c 1}, A_{c 2}, \ldots, A_{c M}\right\}$, where the matrices $A_{c p}$ are asymptotically stable and commute pairwise. Then, we get the following:

(1) The system is exponentially stable under any arbitrary switching between the elements of $A_{c}$.

(2) There exists a common Lyapunov function for all the subsystems. For any positive symmetric definite matrix $P_{c 0}$, let $P_{c 1}, P_{c 2}, \ldots, P_{c M}$ be the unique symmetric positive definite solutions to the Lyapunov equations

$$
A_{c p}^{T} P_{c p}+P_{c p} A_{c p}=-P_{c p-1}, \quad c p=c 1, c 2, \ldots, c M
$$

The function $V(x)=x^{T} P_{c M} x$ is a common Lyapunov function for each of the individual system $\dot{x}=A_{c} x(t)$ and hence a Lyapunov function for the switching system.

Remark 9. All of the proposed descriptions and notations are identical to the given multisystem.

Theorem 10 (see [1]). Consider a classical mixed switched system composed of continuous-time subsystems $\dot{x}=A_{c} x(t)$ with $A_{c} \triangleq\left\{A_{c 1}, A_{c 2}, \ldots, A_{c M}\right\}$ and discrete-time subsystems $x(k+1)=A_{d} x(k)$ with $A_{d} \triangleq\left\{A_{d 1}, A_{d 2}, \ldots, A_{d M}\right\}$. Let $A_{c p}$ be Hurwitz stable and $A_{d q}$ be Schur stable to commute pairwise. Then, we get the following:

(1) The system is exponentially stable for any arbitrary switching between the elements of $A$.

(2) There exists a common Lyapunov function for all the subsystems. For any positive definite matrix $P_{d 0}$, let $P_{d 1}, P_{d 2}, \ldots, P_{d M}$ be the unique positive definite solutions to the Lyapunov equations

$$
\begin{aligned}
& A_{d 1}^{T} P_{d 1} A_{d 1}+P_{d 1}=-P_{d 0} \\
& A_{d q}^{T} P_{d q} A_{d q}+P_{d q}=-P_{d q-1}
\end{aligned}
$$

let $P_{c 1}, P_{c 2}, \ldots, P_{c M}$ be the unique positive definite solutions to the Lyapunov equations

$$
A_{c p}^{T} P_{c p}+P_{c p} A_{c p}=-P_{c p-1}
$$

$$
A_{c 1}^{T} P_{c 1}+P_{c 1} A_{d 1}=-P_{d M}
$$

The function $V(x)=x^{T} P_{c M} x$ is a common Lyapunov function for each of the individual system $\dot{x}=A_{c} x(t), x(k+$ $1)=A_{d} x(k)$ and hence a Lyapunov function for the switching system.

Remark 11. Same as Remark 9.

Clearly, in Theorems 8 and 10, as a necessary condition, all the subsystems that are asymptotically stable still cannot ensure a stable switched system under arbitrary switching. Then, the sufficient condition is needed that is pairwise commutative, which guarantees the stability of the switched system. In other words, if there exists a common Lyapunov function for all the subsystems, the stability is ensured under arbitrary switching, and the solutions are gained.

In the following section, matrices $E$ is analysed, and some lemmas and theories are derived. The exponential stability of the system under any arbitrary switching is discussed, and a common Lyapunov function can be obtained.

\section{Main Results}

In the framework of proposed multiswitched system, and based on Theorems 8 and 10, we have the following lemmas.

Lemma 12. If matrices $A_{c}$ and $A_{d}$ are pairwise commutative, matrices $E_{c}$ and $E_{d}$ are also pairwise commutative.

Proof. Consider $A_{c i} A_{c i^{\prime}}=A_{c i^{\prime}} A_{c i}$ of continuous-time subsystems, $i \neq i^{\prime}$.

We get

$$
\begin{aligned}
& E_{c i} E_{c i^{\prime}}=E_{c i^{\prime}} E_{c i} \\
& =\left(\begin{array}{ccccc}
A_{c 1} & \cdots & 0 & \cdots & 0 \\
\vdots & \ddots & & & \vdots \\
0 & & A_{c p} & & 0 \\
\vdots & & & \ddots & \vdots \\
0 & \cdots & 0 & \cdots & A_{c M}
\end{array}\right)\left(\begin{array}{ccccc}
A_{c 1} & \cdots & 0 & \cdots & 0 \\
\vdots & \ddots & & & \vdots \\
0 & & A_{c p} & & 0 \\
\vdots & & & \ddots & \vdots \\
0 & \cdots & 0 & \cdots & A_{c M}
\end{array}\right)
\end{aligned}
$$

Consider $A_{c} A_{d}=A_{d} A_{c}$ of all subsystems; we get

$$
\begin{aligned}
& E_{c} E_{d}=\left(\begin{array}{ccccc}
A_{c 1} & \cdots & 0 & \cdots & 0 \\
\vdots & \ddots & & & \vdots \\
0 & & A_{c p} & & 0 \\
\vdots & & & \ddots & \vdots \\
0 & \cdots & 0 & \cdots & A_{c M}
\end{array}\right)\left(\begin{array}{ccccc}
A_{d 1} & \cdots & 0 & \cdots & 0 \\
\vdots & \ddots & & & \vdots \\
0 & & A_{d q} & & 0 \\
\vdots & & & \ddots & \vdots \\
0 & \cdots & 0 & \cdots & A_{d M}
\end{array}\right)=\left(\begin{array}{ccccc}
A_{c 1} A_{d 1} & \cdots & 0 & \cdots & 0 \\
\vdots & \ddots & & \vdots \\
0 & & A_{c p} A_{d p} & 0 \\
\vdots & & & \ddots & \vdots \\
0 & \cdots & 0 & \cdots & A_{c M} A_{d M}
\end{array}\right) \\
& =\left(\begin{array}{ccccc}
A_{d 1} A_{c 1} & \cdots & 0 & \cdots & 0 \\
\vdots & \ddots & & & \vdots \\
0 & & A_{d q} A_{c p} & & 0 \\
\vdots & & & \ddots & \vdots \\
0 & \cdots & 0 & \cdots & A_{d M} A_{c M}
\end{array}\right)=E_{d} E_{c}
\end{aligned}
$$


$E_{d i} E_{d i^{\prime}}=E_{d i^{\prime}} E_{d i}$ is similar with (11).

Therefore, the matrices $E_{c}$ and $E_{d}$ are pairwise commutative. This completes the proof for Lemma 12.

Lemma 13. If matrices $A_{c}$ and $A_{d}$ are Hurwitz stable and Schur stable, respectively, matrices $E_{c}$ and $E_{d}$ are also Hurwitz stable and Schur stable, respectively.

Proof. According to the Hurwitz stable criterion, all the order principal minors of matrices $E$ must be positive.

The structure of (2) is parallel, and matrices $E$ are diagonal. Thus, all the order principal minors are also diagonal. $A_{c p}, c p=c 1, \ldots, c M$ are Hurwitz stable, and all the order principal minors are positive; thus, it is proven that matrices $E$ are Hurwitz stable.

$E_{d}$ is Schur stable, which is similar with the Hurwitz stable criterion.

Remark 14. Unnecessary zero rows or zero columns can be omitted.

Based on Theorem 8 and Lemmas 12 and 13, we have the following results.

Proposition 15. Assume $\dot{x}=A_{c} x(t)$ are Hurwitz stable and $A_{c p}$ are pairwise commutative. Then, we get the following:

(1) The continuous-time multiswitched system is exponentially stable for any arbitrary switching between the elements of $E_{c}$.

(2) There exists a common Lyapunov function for all the subsystems-groups and subsystems. For any positive symmetric definite matrix $P_{c 0}$, let $P_{c 1}, P_{c 2}, \ldots, P_{c M}$ be the unique symmetric positive definite solutions to the Lyapunov equations

$$
\begin{aligned}
& \left(A_{c 1}^{T} P_{i}+P_{i} A_{c 1}\right)+\left(A_{c 2}^{T} P_{i}+P_{i} A_{c 2}\right)+\cdots \\
& +\left(A_{c M}^{T} P_{i}+P_{i} A_{c M}\right)=-P_{i-1}, \\
& \quad c p=c 1, c 2, \ldots, c M ; i=1,2, \ldots, N_{c}
\end{aligned}
$$

The function $V(x)=x^{T} P_{N_{c}} x$ is a common Lyapunov function for each of the individual systems $\dot{x}=A_{c} x(t)$ and is thus a Lyapunov function for the switching system.

Proof. Based on Lemmas 12 and 13, $E_{c i}$ are pairwise commutative, and $E_{c}$ are Hurwitz stable. Combining Theorem 8, we get

$$
E_{c i}^{T} P_{i}+P_{i} E_{c i}=-P_{i-1}, \quad i=1, \ldots, N_{c}
$$

We substitute $E_{c i}$ in (2) into (14) to obtain

$$
\left(\begin{array}{ccccc}
A_{c 1} & \cdots & 0 & \cdots & 0 \\
\vdots & \ddots & & & \vdots \\
0 & & A_{c p} & & 0 \\
\vdots & & & \ddots & \vdots \\
0 & \cdots & 0 & \cdots & A_{c M}
\end{array}\right)^{T} P_{i}
$$

$$
+P_{i}\left(\begin{array}{ccccc}
A_{c 1} & \cdots & 0 & \cdots & 0 \\
\vdots & \ddots & & & \vdots \\
0 & & A_{c p} & & 0 \\
\vdots & & & \ddots & \vdots \\
0 & \cdots & 0 & \cdots & A_{c M}
\end{array}\right)=-P_{i-1}
$$

Equation (15) equals (14), which implies that the system is exponentially stable for arbitrary switching; (15) transforms into (13), which implies the solutions to the Lyapunov equations.

Example 16. Consider a set $A_{c}=\left\{A_{c 1}, A_{c 2}, A_{c 3}, A_{c 4}, A_{c 5}\right\}$ of a switched system, which are the constant matrices of 5 continuous-time subsystems. The multiswitched system switches between subsystems-groups $E_{c}$. Here, we select only three subsystems-groups as an example.

Let $E_{c}=\left\{E_{1}, E_{2}, E_{3}\right\}, E_{1}=\left[A_{c 1}\right], E_{2}=\left[\begin{array}{cc}A_{c 2} & 0 \\ 0 & A_{c 3}\end{array}\right]$, and $E_{3}=\left[\begin{array}{ccc}A_{c 3} & 0 & 0 \\ 0 & A_{c 4} & 0 \\ 0 & 0 & A_{c 5}\end{array}\right]$

Then, for any positive symmetric definite matrix $P_{c 0}$, there are $P_{c 1}, P_{c 2}$, and $P_{c 3}$ as the unique symmetric positive definite solutions to the Lyapunov equations

$$
\begin{aligned}
& E_{c 1}^{T} P_{1}+P_{1} E_{c 1}=-P_{c 0} \\
& E_{c 2}^{T} P_{2}+P_{2} E_{c 2}=-P_{c 1} \\
& E_{c 3}^{T} P_{3}+P_{3} E_{c 3}=-P_{c 2}
\end{aligned}
$$

Equations (16a), (16b), and (16c) can be rewritten as

$$
\begin{aligned}
& A_{c 1}^{T} P_{1}+P_{1} A_{c 1}=-P_{c 0} \\
& A_{c 2}^{T} P_{2}+P_{2} A_{c 2}+A_{c 3}^{T} P_{2}+P_{2} A_{c 3}=-P_{c 1} \\
& A_{c 3}^{T} P_{3}+P_{3} A_{34}+A_{c 4}^{T} P_{3}+P_{3} A_{c 4}+A_{c 5}^{T} P_{3}+P_{3} A_{c 5} \\
& \quad=-P_{c 2}
\end{aligned}
$$

The function $V(x)=x^{T} P_{3} x$ is a common Lyapunov function for each of the individual system $\dot{x}=A_{c} x(t)$ with $c p=c 1, c 2, c 3, c 4$, and $c 5$.

Remark 17. The above theorem and proof can be extended to the case of discrete-time multiswitched system $x(k+1)=$ $E_{d j} x(k)$ with $j=1, \ldots, N_{d}$. Assume the matrices $x(k+$ $1)=A_{d q} x(k)$ with $d q=d 1, \ldots, d M$ are Schur stable and commute pairwise. Then, the discrete-time multiswitched system is exponentially stable for arbitrary switching between the elements of $E_{d}$. The solution (13) can be modified as

$$
\begin{aligned}
& A_{d 1}^{T} P_{j} A_{d 1}+A_{d 2}^{T} P_{j} A_{d 2}+\cdots=A_{d M}^{T} P_{j} A_{d M} \\
& \quad=P_{j}-P_{j-1}, \quad d q=d 1, d 2, \ldots, d M ; j=1, \ldots, N_{d}
\end{aligned}
$$

and the common Lyapunov function is $V(x)=x^{T} P_{N_{d}} x$. 
Proposition 18. Assume $\dot{x}=A_{c} x(t)$ are Hurwitz stable and $x(k+1)=A_{d} x(k)$ are Schur stable; $A_{c p}$ and $A_{d q}$ are pairwise commutative. Then, we get the following:

(1) The multiswitched system is exponentially stable for any arbitrary switching between the elements of $E$.

(2) There exists a common Lyapunov function for all the subsystems. For any positive symmetric definite matrix $P_{d 0}$, let $P_{d 1}, P_{d 2}, \ldots, P_{d N d}$ be the unique positive definite solutions to the Lyapunov equations

$$
\begin{gathered}
A_{d 1}^{T} P_{d 1} A_{d 1}+\cdots+A_{d M}^{T} P_{d 1} A_{d M}-P_{d 1}=-P_{d 0}, \\
d q=d 1, d 2, \ldots, d M \\
A_{d 1}^{T} P_{d j} A_{d 1}+\cdots+A_{d M}^{T} P_{d j} A_{d M}-P_{d j}=-P_{d j-1}, \\
d q=d 1, d 2, \ldots, d M ; j=1, \ldots, N_{d}
\end{gathered}
$$

Let $P_{c 1}, P_{c 2}, \ldots, P_{c N}$ be the unique positive definite solutions to the Lyapunov equations

$$
\begin{aligned}
& \left(A_{c 1}^{T} P_{c 1}+P_{c 1} A_{d 1}\right)+\cdots+\left(A_{c M}^{T} P_{c 1}+P_{c 1} A_{d M}\right) \\
& =-P_{d N d}, \\
& \quad c p=c 1, c 2, \ldots, c M ; d q=d 1, d 2, \ldots, d M \\
& \left(A_{c 1}^{T} P_{c i}+P_{c i} A_{c 1}\right)+\cdots+\left(A_{c M}^{T} P_{c i}+P_{c i} A_{c M}\right) \\
& =-P_{c i-1}, \quad c p=c 1, c 2, \ldots, c M ; i=1,2, \ldots, N_{c}
\end{aligned}
$$

The function $V(x)=x^{T} P_{c N_{c}} x$ is a common Lyapunov function for each of the individual system $\dot{x}=A_{c} x(t)$, and $x(k+1)=A_{d q} x(k)$; hence a Lyapunov function is used for the switching system.

Proof. Based on Theorem 10 as well as Lemmas 12 and 13, we get

$$
\begin{aligned}
E_{d 1}^{T} P_{d 1} E_{d 1}-P_{d 1} & =-P_{d 0} \\
E_{d j}^{T} P_{d j} E_{d j}-P_{d j} & =-P_{d j-1} \\
E_{c 1}^{T} P_{c 1}+P_{c 1} E_{d 1} & =-P_{d N d} \\
E_{c i}^{T} P_{c i}+P_{c i} E_{c i} & =-P_{c i-1}
\end{aligned}
$$

We substitute $E_{c i}$ and $E_{d j}$ in (2) into (19a), (19b), (19c), and (19d) to obtain

$$
\left(\begin{array}{ccccc}
A_{d 1} & \cdots & 0 & \cdots & 0 \\
\vdots & \ddots & & & \vdots \\
0 & & A_{d q} & & 0 \\
\vdots & & & \ddots & \vdots \\
0 & \cdots & 0 & \cdots & A_{d M}
\end{array}\right)^{T}
$$

$$
\begin{gathered}
\cdot P_{d 1}\left(\begin{array}{ccccc}
A_{d 1} & \cdots & 0 & \cdots & 0 \\
\vdots & \ddots & & & \vdots \\
0 & & A_{d q} & & 0 \\
\vdots & & & \ddots & \vdots \\
0 & \cdots & 0 & \cdots & A_{d M}
\end{array}\right)-P_{d 1} \\
=-P_{d 0}
\end{gathered}
$$

$$
\left(\begin{array}{ccccc}
A_{d 1} & \cdots & 0 & \cdots & 0 \\
\vdots & \ddots & & & \vdots \\
0 & & A_{d q} & & 0 \\
\vdots & & & \ddots & \vdots \\
0 & \cdots & 0 & \cdots & A_{d M}
\end{array}\right)^{T}
$$$$
\cdot P_{d j}\left(\begin{array}{ccccc}
A_{d 1} & \cdots & 0 & \cdots & 0 \\
\vdots & \ddots & & & \vdots \\
0 & & A_{d q} & & 0 \\
\vdots & & & \ddots & \vdots \\
0 & \cdots & 0 & \cdots & A_{d M}
\end{array}\right)-P_{d j}
$$

$$
=-P_{d j-1}
$$

$$
\begin{gathered}
\left(\begin{array}{ccccc}
A_{c 1} & \cdots & 0 & \cdots & 0 \\
\vdots & \ddots & & & \vdots \\
0 & & A_{c p} & & 0 \\
\vdots & & & \ddots & \vdots \\
0 & \cdots & 0 & \cdots & A_{c M}
\end{array}\right){ }^{T} P_{c 1} \\
+P_{c 1}\left(\begin{array}{ccccc}
A_{d 1} & \cdots & 0 & \cdots & 0 \\
& \vdots & \ddots & & \\
0 & & A_{d q} & & 0 \\
\vdots & & & \ddots & \vdots \\
0 & \cdots & 0 & \cdots & A_{d M}
\end{array}\right)=-P_{d N d}
\end{gathered}
$$


$$
+P_{c i}\left(\begin{array}{ccccc}
A_{c 1} & \cdots & 0 & \cdots & 0 \\
\vdots & \ddots & & & \vdots \\
0 & & A_{c p} & & 0 \\
\vdots & & & \ddots & \vdots \\
0 & \cdots & 0 & \cdots & A_{c M}
\end{array}\right)=-P_{c i-1}
$$

Equations (21a), (21b), (21c), and (21d) equal (20a), (20b), (20c), and (20d), which implies that the system is exponentially stable for any arbitrary switching; and (21a), (21b), (21c), and (21d) transform into (19a), (19b), (19c), and (19d), which implies the solutions to the Lyapunov equations.

Example 19. Consider sets $A_{c}=\left\{A_{c 1}, A_{c 2}, A_{c 3}\right\}$ and $A_{d}=$ $\left\{A_{d 1}, A_{d 2}\right\}$ of a switched system, which are the constant matrices of 3 continuous-time subsystems and 2 discrete-time subsystems, respectively. The multiswitched system switches between in subsystems-groups $E$. Here, we select only five subsystems-groups as an example.

Let $E_{c}=\left\{E_{c 1}, E_{c 2}, E_{c 3}\right\}, E_{c 1}=\left[\begin{array}{cc}A_{c 1} & 0 \\ 0 & A_{c 3}\end{array}\right], E_{c 2}=\left[\begin{array}{cc}A_{c 2} & 0 \\ 0 & A_{c 3}\end{array}\right]$, and $E_{c 3}=\left[\begin{array}{ccc}A_{c 1} & 0 & 0 \\ 0 & A_{c 2} & 0 \\ 0 & 0 & A_{c 3}\end{array}\right]$; let $E_{d}=\left\{E_{d 1}, E_{d 2}\right\}, E_{d 1}=\left[A_{d 2}\right]$, and $E_{d 2}=\left[\begin{array}{cc}A_{d 1} & 0 \\ 0 & A_{d 2}\end{array}\right]$.

Then, for any positive definite matrix $P_{d 0}$, there are $P_{d 1}$, $P_{d 2}, P_{c 1}, P_{c 2}$, and $P_{c 3}$ as the unique symmetric positive definite solutions to the Lyapunov equations

$$
\begin{aligned}
& E_{d 1}^{T} P_{d 1} E_{d 1}-P_{d 1}=-P_{d 0} \\
& E_{d 2}^{T} P_{d 2} E_{d 2}-P_{d 2}=-P_{d 1} \\
& E_{c 1}^{T} P_{c 1}+P_{c 1} E_{d 1}=-P_{d 2} \\
& E_{c 2}^{T} P_{c 2}+P_{c 2} E_{c 2}=-P_{c 1} \\
& E_{c 3}^{T} P_{c 3}+P_{c 3} E_{c 3}=-P_{c 2}
\end{aligned}
$$

Equations (22a), (22b), (22c), (22d), and (22e) can be rewritten as

$$
\begin{aligned}
& A_{d 2}^{T} P_{d 1} A_{d 2}-P_{d 1}=-P_{d 0} \\
& A_{d 1}^{T} P_{d 2} A_{d 1}+A_{d 2}^{T} P_{d 2} A_{d 2}-P_{d 2}=-P_{d 1} \\
& A_{c 1}^{T} P_{c 1}+A_{c 2}^{T} P_{c 1}+P_{c 1} A_{d 2}=-P_{d 2} \\
& A_{c 2}^{T} P_{c 2}+P_{c 2} A_{c 2}+A_{c 3}^{T} P_{c 2}+P_{c 2} A_{c 3}=-P_{c 1} \\
& A_{c 1}^{T} P_{c 3}+P_{c 3} A_{c 1}+A_{c 2}^{T} P_{c 3}+P_{c 3} A_{c 2}+A_{c 3}^{T} P_{c 3} \\
& \quad+P_{c 3} A_{c 3}=-P_{c 2}
\end{aligned}
$$

The function $V(x)=x^{T} P_{c 3} x$ is a common Lyapunov function for each of the individual systems $\dot{x}=A_{c} x(t)$ with $c p=c 1, c 2, c 3$ and $x(k+1)=A_{d} x(k)$ with $d q=d 1, d 2$, respectively.

\section{Results in Parallel-Like Structures}

Lemma 20. If the structures of $E_{c}$ are not standard parallel and contain coupled components based on a parallel framework (i.e., the subsystems are not independent of each other), then the matrices should be modified as

$$
E_{c i}=\left(\begin{array}{ccccc}
A_{c 1} & \cdots & A_{o(1, p)} & \cdots & A_{o(1, c M)} \\
\vdots & \ddots & & & \vdots \\
A_{o(p, 1)} & & A_{c p} & & A_{o(p, c M)} \\
\vdots & & & \ddots & \vdots \\
A_{o(c M, 1)} & \cdots & A_{o(c M, p)} & \cdots & A_{c M}
\end{array}\right)
$$

$A_{o\left(p, p^{\prime}\right)}$ are matrices of the coupled components, $p=$ $c 1, \ldots, c M$, and $p \neq p^{\prime}$. Clearly, there are another nonzero elements except in the main diagonal line.

Remark 21. Considering the coupled components, $E_{d}$ can be modified as

$$
E_{d j}=\left(\begin{array}{ccccc}
A_{d 1} & \cdots & A_{o(1, q)} & \cdots & A_{o(1, d M)} \\
\vdots & \ddots & & & \vdots \\
A_{o(q, 1)} & & A_{d q} & & A_{o(q, d M)} \\
\vdots & & & \ddots & \vdots \\
A_{o(d M, 1)} & \cdots & A_{o(d M, q)} & \cdots & A_{d M}
\end{array}\right)
$$

$A_{o\left(q, q^{\prime}\right)}$ are matrices of the coupled components, $q=$ $q 1, \ldots, q M$, and $q \neq q^{\prime}$.

Remark 22. The structures proposed in Lemma 20 and Remark 21 are named as parallel-like. Only the coupled component is the distinction of structure between parallel and parallel-like; in other words, the parallel-like structure is minor alteration on the parallel structure.

Proposition 23. Under the assumption of Lemma 20, the framework of $E_{c}$ in Lemma 20 can be classified as the following 3 types: upper triangular, lower triangular, and other nonregular modes. Then, Proposition 15 can be modified as follows:

(a) If the framework of $E_{c}$ is upper triangular, Proposition 15 is true, but the solutions is 


$$
\left(\begin{array}{ccccc}
A_{c 1}^{T} P_{i}+P_{i} A_{c 1} & \cdots & P_{i} A_{o(1, p)} & \cdots & P_{i} A_{o(1, c M)} \\
\vdots & \ddots & & & \vdots \\
A_{o(1, p)}^{T} P_{i} & & A_{c p}^{T} P_{i}+P_{i} A_{c p} & & P_{i} A_{o(p, c M)} \\
\vdots & & & \ddots & \vdots \\
A_{o(1, c M)}^{T} P_{i} & \cdots & A_{o(p, c M)}^{T} P & \cdots & A_{c M}^{T} P_{i}+P_{i} A_{c M}
\end{array}\right)=-P_{i-1}
$$

where $c p=c 1, c 2, \ldots, c M ; i=1,2, \ldots, N c$. The function $V(x)=x^{T} P_{N_{c}} x$ is a common Lyapunov function for each of the individual system $\dot{x}=A_{c} x(t)$. (b) Lower triangular is same as upper triangular. The solution is

$$
\left(\begin{array}{ccccc}
A_{c 1}^{T} P_{i}+P_{i} A_{c 1} & \cdots & A_{o(p, 1)}^{T} P_{i} & \cdots & A_{o(c M, 1)}^{T} P_{i} \\
\vdots & \ddots & & & \vdots \\
P_{i} A_{o(1, p)} & & A_{c p}^{T} P_{i}+P_{i} A_{c p} & & A_{o(c M, p)}^{T} P_{i} \\
\vdots & & & \ddots & \vdots \\
P_{i} A_{o(1, c M)} & \cdots & P_{i} A_{o(p, c M)} & \cdots & A_{c M}^{T} P_{i}+P_{i} A_{c M}
\end{array}\right)=-P_{i-1}
$$

(c) Other nonregular modes must satisfy Hurwitz stable, only this, which have ideal stability. The solution is

$$
\left(\begin{array}{ccccc}
A_{c 1}^{T} P_{i}+P_{i} A_{c 1} & \cdots & A_{o(p, 1)}^{T} P_{i}+P_{i} A_{o(1, p)} & \cdots & A_{o(c M, 1)}^{T} P_{i}+P_{i} A_{o(1, c M)} \\
\vdots & \ddots & & & \vdots \\
A_{o(1, p)}^{T} P_{i}+P_{i} A_{o(1, p)} & & A_{c p}^{T} P_{i}+P_{i} A_{c p} & & A_{o(c M, p)}^{T} P_{i}+P_{i} A_{o(p, c M)} \\
\vdots & & \ddots & \vdots \\
A_{o(1, c M)}^{T} P+P_{i} A_{o(1, c M)} & \cdots & A_{o(p, c M)}^{T} P+P_{i} A_{o(p, c M)} & \cdots & A_{c M}^{T} P_{i}+P_{i} A_{c M}
\end{array}\right)=-P_{i-1}
$$

Proof. The condition of $E_{c}$ that is pairwise commutative is determined as follows:

$$
E_{c i} E_{c i^{\prime}}=E_{c i^{\prime}} E_{c i}=\left(\begin{array}{ccccc}
A_{c 1} & \cdots & A_{o(1, p)} & \cdots & A_{o(1, c M)} \\
\vdots & \ddots & & & \vdots \\
A_{o(p, 1)} & & A_{c p} & & A_{o(p, c M)} \\
\vdots & & & \ddots & \vdots \\
A_{o(c M, 1)} & \cdots & A_{o(c M, p)} & \cdots & A_{c M}
\end{array}\right)\left(\begin{array}{ccccc}
A_{c 1} & \cdots & A_{o(1, p)} & \cdots & A_{o(1, c M)} \\
\vdots & \ddots & & \vdots \\
A_{o(p, 1)} & & A_{c p} & A_{o(p, c M)} \\
\vdots & & \ddots & \vdots \\
A_{o(c M, 1)} & \cdots & A_{o(c M, p)} & \cdots & A_{c M}
\end{array}\right)
$$

The Hurwitz stability of $E_{c}$ can be ensured by the framework of upper triangular, in which all the order principal minors of matrices are positive, if $A_{c i}$ is
Hurwitz stable. It satisfies the Hurwitz stable criterion, which is the same as the framework of the lower triangular. 
Thus, the two frameworks of a continuous-time multiswitched system are exponentially stable for any arbitrary switching between the elements of $E_{c}$. There exists a common Lyapunov function for all the subsystems-groups and subsystems. In the framework of the upper triangular, we modify (14) as follows:

$$
\begin{gathered}
\left(\begin{array}{ccccc}
A_{c 1} & \cdots & A_{o(1, p)} & \cdots & A_{o(1, c M)} \\
\vdots & \ddots & & & \vdots \\
0 & & 0 & & A_{o(p, c M)} \\
\vdots & & & \ddots & \vdots \\
0 & \cdots & 0 & \cdots & A_{c M}
\end{array}\right) P^{T} \\
+P_{i}\left(\begin{array}{ccccc}
A_{c 1} & \cdots & A_{o(1, p)} & \cdots & A_{o(1, c M)} \\
\vdots & \ddots & & & \vdots \\
0 & & A_{c p} & & A_{o(p, c M)} \\
\vdots & & & \ddots & \vdots \\
0 & \cdots & 0 & \cdots & A_{c M}
\end{array}\right) \\
=-P_{i-1} \\
\end{gathered}
$$

Obviously, (28) transforms into (26a), which implies the solutions to the Lyapunov equations.

It is similar with upper triangular, in the framework of the lower triangular, we modify (14) as

$$
\begin{aligned}
& \left(\begin{array}{ccccc}
A_{c 1} & \cdots & 0 & \cdots & 0 \\
\vdots & \ddots & & & \vdots \\
A_{o(p, 1)} & & A_{c p} & & 0 \\
\vdots & & & \ddots & \vdots \\
A_{o(c M, 1)} & \cdots & A_{o(c M, p)} & \cdots & A_{c M}
\end{array}\right)^{T} P_{i} \\
& +P_{i}\left(\begin{array}{ccccc}
A_{c 1} & \cdots & 0 & \cdots & 0 \\
\vdots & \ddots & & & \vdots \\
A_{o(p, 1)} & & A_{c p} & & 0 \\
\vdots & & & \ddots & \vdots \\
A_{o(c M, 1)} & \cdots & A_{o(c M, p)} & \cdots & A_{c M}
\end{array}\right) \\
& =-P_{i-1}
\end{aligned}
$$

Of course, (29) transforms into (26b), which implies the solutions to the Lyapunov equations.

However, in other nonregular modes, the stability cannot be guaranteed. The framework is nonregular, so the $E_{c}$ Hurwitz needs to be stable. Then, the system stability under arbitrary switching is ensured, and a common Lyapunov function can be gained as (26c).
Remark 24. The above theorem and proof can be extended to the structure (see (25)) of the discrete-time multiswitched system $x(k+1)=E_{d j} x(k)$ with $j=1, \ldots, N_{d}$. Remark 17 can be modified as follows:

(a) If the framework of $E_{d}$ is an upper triangular, Remark 17 is true; however, the solutions are

$$
\begin{gathered}
\left(\begin{array}{ccccc}
A_{d 1} & \cdots & A_{o(1, q)} & \cdots & A_{o(1, d M)} \\
\vdots & \ddots & & & \vdots \\
0 & & A_{d q} & & A_{o(q, d M)} \\
\vdots & & & \ddots & \vdots \\
0 & \cdots & 0 & \cdots & A_{d M}
\end{array}\right)^{T} \\
\cdot\left(\begin{array}{ccccc}
A_{d 1} & \cdots & A_{o(1, q)} & \cdots & A_{o(1, d M)} \\
\vdots & \ddots & & & \vdots \\
0 & & A_{d q} & & A_{o(q, d M)} \\
\vdots & & & \ddots & \vdots \\
0 & \cdots & 0 & \cdots & A_{d M}
\end{array}\right)=P_{i}
\end{gathered}
$$

where $d q=d 1, d 2, \ldots, d M ; j=1,2, \ldots, N d$. The function $V(x)=x^{T} P_{N_{d}} x$ is a common Lyapunov function for each of the individual systems $\dot{x}=A_{d} x(t)$.

(b) Lower triangular is same as upper triangular. The solution is

$$
\begin{aligned}
& \left(\begin{array}{ccccc}
A_{d 1} & \cdots & 0 & \cdots & 0 \\
\vdots & \ddots & & & \vdots \\
A_{o(q, 1)} & & A_{d q} & & 0 \\
\vdots & & & \ddots & \vdots \\
A_{o(d M, 1)} & \cdots & A_{o(d M, q)} & \cdots & A_{d M}
\end{array}\right)^{T} \\
& \cdot P_{j}\left(\begin{array}{ccccc}
A_{d 1} & \cdots & 0 & \cdots & 0 \\
\vdots & \ddots & & & \vdots \\
A_{o(q, 1)} & & A_{d q} & & 0 \\
\vdots & & & \ddots & \vdots \\
A_{o(d M, 1)} & \cdots & A_{o(d M, q)} & \cdots & A_{d M}
\end{array}\right) \\
& =P_{j}-P_{j-1}
\end{aligned}
$$

(c) Other nonregular modes must satisfy Schur stable, which has ideal stability. The solution is 


$$
\begin{gathered}
\left(\begin{array}{ccccc}
A_{d 1} & \cdots & A_{o(1, q)} & \cdots & A_{o(1, d M)} \\
\vdots & \ddots & & & \vdots \\
A_{o(q, 1)} & & A_{d q} & & A_{o(q, d M)} \\
\vdots & & & \ddots & \vdots \\
A_{o(d M, 1)} & \cdots & A_{o(d M, q)} & \cdots & A_{d M}
\end{array}\right)^{T} \\
\cdot\left(\begin{array}{ccccc}
A_{d 1} & \cdots & A_{o(1, q)} & \cdots & A_{o(1, d M)} \\
\vdots & \ddots & & & \vdots \\
A_{o(q, 1)} & & A_{d q} & & A_{o(q, d M)} \\
\vdots & & & \ddots & \vdots \\
A_{o(d M, 1)} & \cdots & A_{o(d M, q)} & \cdots & A_{d M}
\end{array}\right) \\
=P_{j}-P_{j-1}
\end{gathered}
$$

If the above theorems and remarks extend to mixed switched system, we find it difficult to get the condition of pairwise commutative, whatever the triangular and other frameworks in $E$. In some special situations, it satisfies the assuming condition $E_{c} E_{d}=E_{d} E_{c}$. Then, the matrices $E_{c}$ and $E_{d}$ should better be in upper or lower triangular framework, to ensure Hurwitz stable and Schur stable, respectively. Otherwise, the stable condition becomes strictly to require matrices $E$ and be unconcerned with matrices $A$.

If all the conditions are satisfied, the goal of stability under arbitrary switching can be gained, and the function $V(x)=x^{T} P_{c N c} x$ is a common Lyapunov function for each of the individual system $\dot{x}=A_{c} x(t)$, and $x(k+$ 1) $=A_{d q} x(k)$. For any positive symmetric definite matrix $P_{d 0}$, thus, $P_{d 1}, P_{d 2}, \ldots, P_{d N d}$ are the unique positive definite solutions to the Lyapunov equations

$$
\begin{gathered}
\left(\begin{array}{ccccc}
A_{d 1} & \cdots & A_{o(1, q)} & \cdots & A_{o(1, d M)} \\
\vdots & \ddots & & & \vdots \\
A_{o(q, 1)} & & A_{d q} & & A_{o(q, d M)} \\
\vdots & & & \ddots & \vdots \\
A_{o(d M, 1)} & \cdots & A_{o(d M, q)} & \cdots & A_{d M}
\end{array}\right)^{T} \\
\cdot\left(\begin{array}{ccccc}
A_{d 1} & \cdots & A_{o(1, q)} & \cdots & A_{o(1, d M)} \\
\vdots & \ddots & & & \vdots \\
A_{o(q, 1)} & & A_{d q} & & A_{o(q, d M)} \\
\vdots & & & \ddots & \vdots \\
A_{o(d M, 1)} & \cdots & A_{o(d M, q)} & \cdots & A_{d M}
\end{array}\right) \\
-P_{d 1}=-P_{d 0}
\end{gathered}
$$
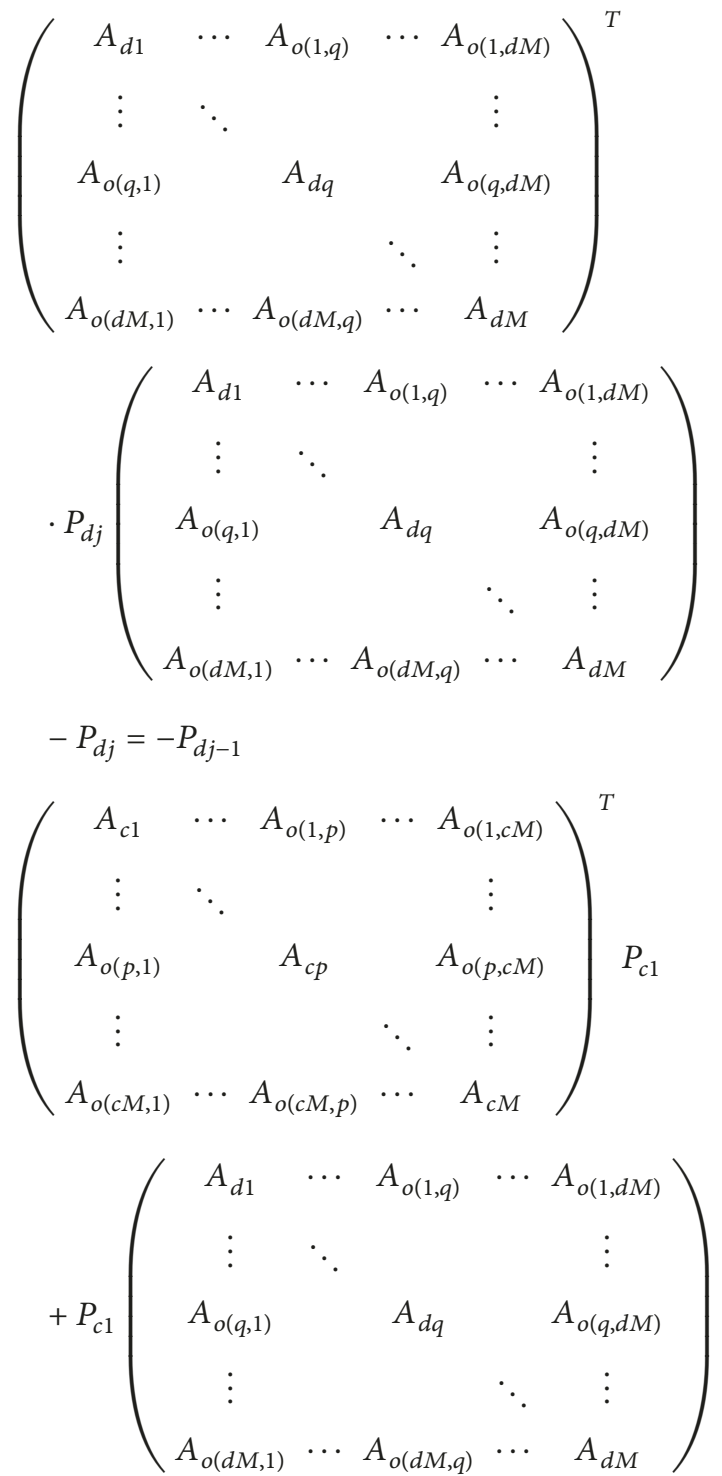

$$
=-P_{d N d}
$$

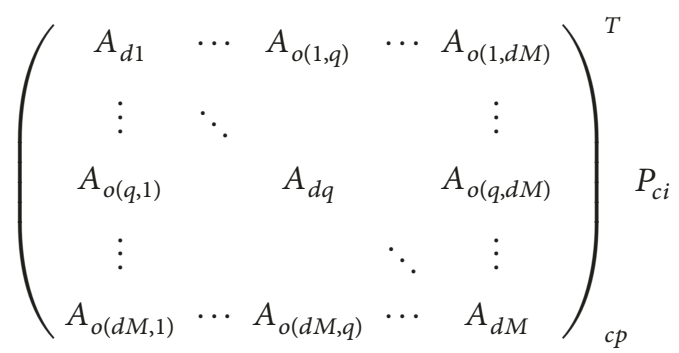

$$
+P_{c i}\left(\begin{array}{ccccc}
A_{d 1} & \cdots & A_{o(1, q)} & \cdots & A_{o(1, d M)} \\
\vdots & \ddots & & & \vdots \\
A_{o(q, 1)} & & A_{d q} & & A_{o(q, d M)} \\
\vdots & & & \ddots & \vdots \\
A_{o(d M, 1)} & \cdots & A_{o(d M, q)} & \cdots & A_{d M}
\end{array}\right)
$$




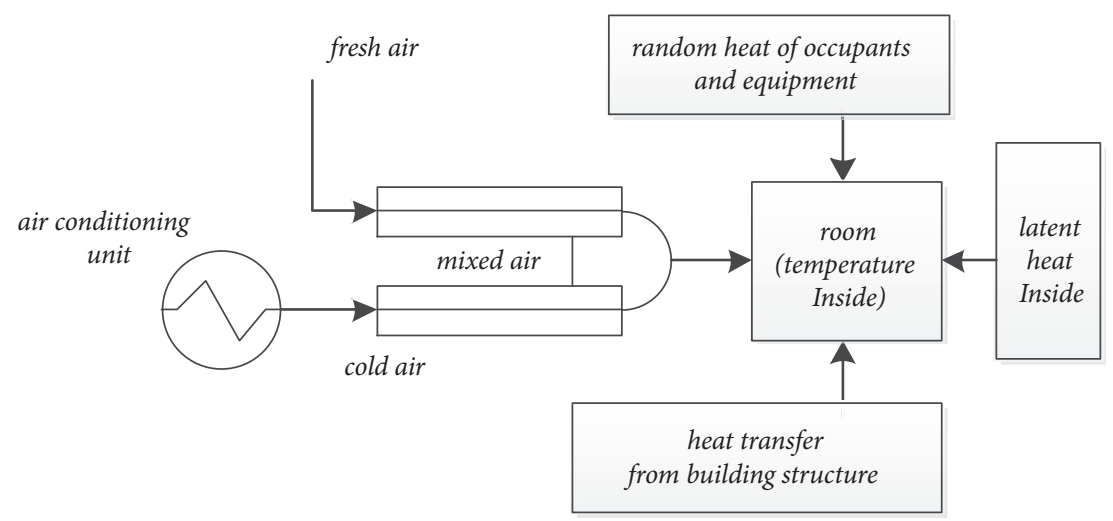

FIgURE 6: Thermal balance of air system.

\section{Numerical Example}

In this section, an engineering application of central air conditioning is introduced as a numerical example, which shows the framework of multiswitched system with parallel structure. In addition, the engineering environment is illustrated and thermal parameters are given in Table 4. Simulation results are presented to illustrate the characteristics of the system and the situation of stability by different control strategies. The mathematical model is given in [27], which shows the thermal balance of a test room affected by factors, such as the structure and materials of a building, outdoor weather parameters, indoor lighting, radiating equipment, and number of occupants (see Figure 6). The cooling capacity is transferred from chilled water system to air system via air conditioning units by the measures of constant air volume and variable water volume/constant temperature difference.

The thermal balance equation is

$$
\begin{aligned}
C_{a} m_{a} \frac{d \theta}{d \tau}= & -\gamma \varepsilon C_{w} \Delta \theta q_{w}-\left(1-R_{r}\right) q_{s a} C_{a} \theta \\
& +\left(1-R_{r}\right) q_{s a} C_{a} \theta_{\text {out }}+Q_{r d}+Q_{q r} \\
& -\sum K_{j} A_{j} \theta+\sum K_{j} A_{j} \theta_{j}
\end{aligned}
$$

where $\theta$ is the real-time indoor temperature. On the left side of the equation, $C_{a} m_{a}(d \theta / d \tau)$ means the time differential of the heat capacity of a room. On the left side of the equation, $-\gamma \varepsilon C_{w} \Delta \theta q_{w}$ means the cooling capacity for chilled water system; $-\left(1-R_{r}\right) q_{s a} C_{a} \theta$ and $\left(1-R_{r}\right) q_{s a} C_{a} \theta_{\text {out }}$ represent the cooling capacity from return and fresh air systems, respectively; $Q_{r d}$ denotes random heat of occupants and equipment; $Q_{q r}$ means latent heat inside; $-\sum K_{j} A_{j} \theta+\sum K_{j} A_{j} \theta_{j}$ is the heat transfer from building structure. The description of the symbols is presented in Table 4 .

A midsize conference room (length: $10 \mathrm{~m}$, width: $6 \mathrm{~m}$, and height: $3 \mathrm{~m}$ ) is simulated, we use two different control strategies (the strategies 1 and 2 mentioned in Examples 6 and 7 , respectively) to adjust cooling capacity and illustrate system stability (corresponding Propositions 15 and 18, respectively) in the framework of multiswitching system with parallel structure, and use strategy 3 to reflect the unstable situation.

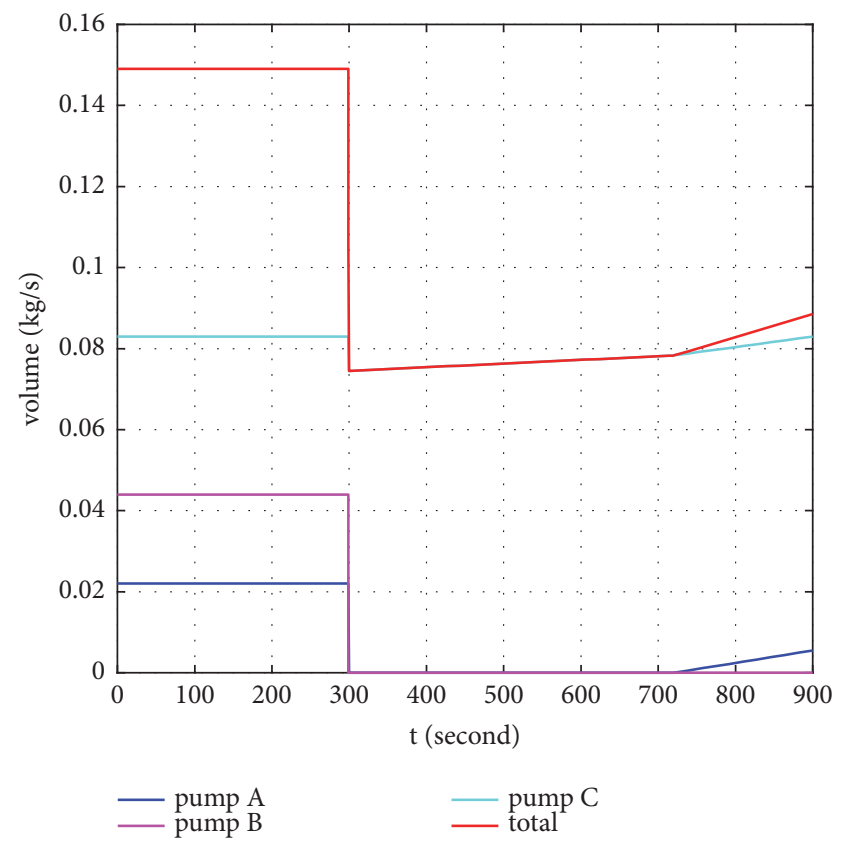

FIGURE 7: Flow volume under strategy 1.

The above three control strategies and the two types of pumps are shown in Table 1 . The range of variable volume is $50 \%$ $100 \%$ and the time is divided into three intervals $([0,5 \mathrm{~min}]$, [ $5 \mathrm{~min}, 12 \mathrm{~min}$ ], and [12 $\mathrm{min}, 15 \mathrm{~min}$ ]) in the above three control strategies. The cold air is sent to the room for reducing the indoor temperature. The indoor temperature is required to be lowered with respect to the initial temperature $\left(\theta_{0}, 30^{\circ} \mathrm{C}\right)$ and regulated at $\left(\theta_{\text {set }}, 26^{\circ} \mathrm{C}\right)$ as soon as possible. In the last 3 minutes, the indoor cooling load increased significantly due to that the number of indoor participants increased (see Figure 10). The outdoor temperature is basically maintained at $30^{\circ} \mathrm{C}$ in the simulated 15 minutes.

Figure 7 shows the switching dynamics of a continuoustime multiswitched system with parallel structure by the flow volume of the three pumps. In the first time interval, all the three pumps work as a subsystems-group in rated volume for reducing the temperature; in the middle time interval, only 
TABLE 1: Control strategies and types of pumps.

\begin{tabular}{cccccc}
\hline strategy & pump & working type & control mode & feedback coefficient & switching state \\
\hline \multirow{4}{*}{1} & $A$ & variable volume & feedback/switching & 0.0074 & ON/OFF \\
& $B$ & variable volume & feedback/switching & NULL & ON/OFF \\
& $C$ & variable volume & feedback/switching & ON & ON/OFF \\
2 & $A$ & fixed volume & switching & NULL & ON/OFF \\
& $B$ & fixed volume & switching & 0.019 & ON \\
& $C$ & variable volume & feedback & NULL & ON/OFF \\
& $A$ & fixed volume & switching & ON/OFF & ON \\
\hline
\end{tabular}

TABLE 2

\begin{tabular}{lcc}
\hline pump & $\begin{array}{c}\text { rated volume } \\
\mathrm{q}_{0}(\mathrm{~kg} / \mathrm{s})\end{array}$ & $\begin{array}{c}\text { range of variable } \\
\text { volume } \\
\mathrm{q}(\mathrm{kg} / \mathrm{s})\end{array}$ \\
\hline $\mathrm{A}$ & 0.01 & $0.005-0.01$ \\
$\mathrm{~B}$ & 0.02 & $0.015-0.02$ \\
$\mathrm{C}$ & 0.033 & $0.0175-0.033$ \\
total & 0.063 & $0.0315-0.063$ \\
\hline
\end{tabular}

TABLE 3

\begin{tabular}{lcc}
\hline pump & $\begin{array}{c}\text { rated volume } \\
\mathrm{q}_{0}(\mathrm{~kg} / \mathrm{s})\end{array}$ & $\begin{array}{c}\text { range of variable } \\
\text { volume } \\
\mathrm{q}(\mathrm{kg} / \mathrm{s})\end{array}$ \\
\hline $\mathrm{A}$ & 0.01 & $0.01-0.01$ \\
$\mathrm{~B}$ & 0.02 & $0.02-0.02$ \\
$\mathrm{C}$ & 0.033 & $0.0175-0.033$ \\
total & 0.063 & $0.0315-0.063$ \\
\hline
\end{tabular}

the pump $C$ works in variable flow mode under the feedback control; in the last time interval, both pump $A$ and pump $C$ work as a subsystems-group under feedback control for creasing cooling capacity because of the increase of indoor participants increased.

Figure 8 shows the switching of a mixed multiswitched system with parallel structure composed of one continuoustime subsystem and two discrete-time subsystems. In the first two time intervals, the dynamics of pumps is the same as Figure 7. In the last time interval, pump $A$ works at the rated volume as a discrete-time subsystem and the pump $C$ works in variable flow mode under feedback control as a continuous-time subsystem; in other words, the subsystemsgroup is composed with one continuous-time and one discrete-time subsystems. Figure 9 is similar to Figure 8, but the switching dynamics is different (in the middle time interval, all the three pumps work together; in the last time interval, only pump $C$ works).

Figure 11 shows the changes of indoor temperature under the three control strategies. The indoor temperature drops from the initial value $\left(30^{\circ} \mathrm{C}\right)$ to the set point $\left(26^{\circ} \mathrm{C}\right)$ in five minutes under the three strategies, because of rated volume by the whole water system. It is worth noting that, in the last 10

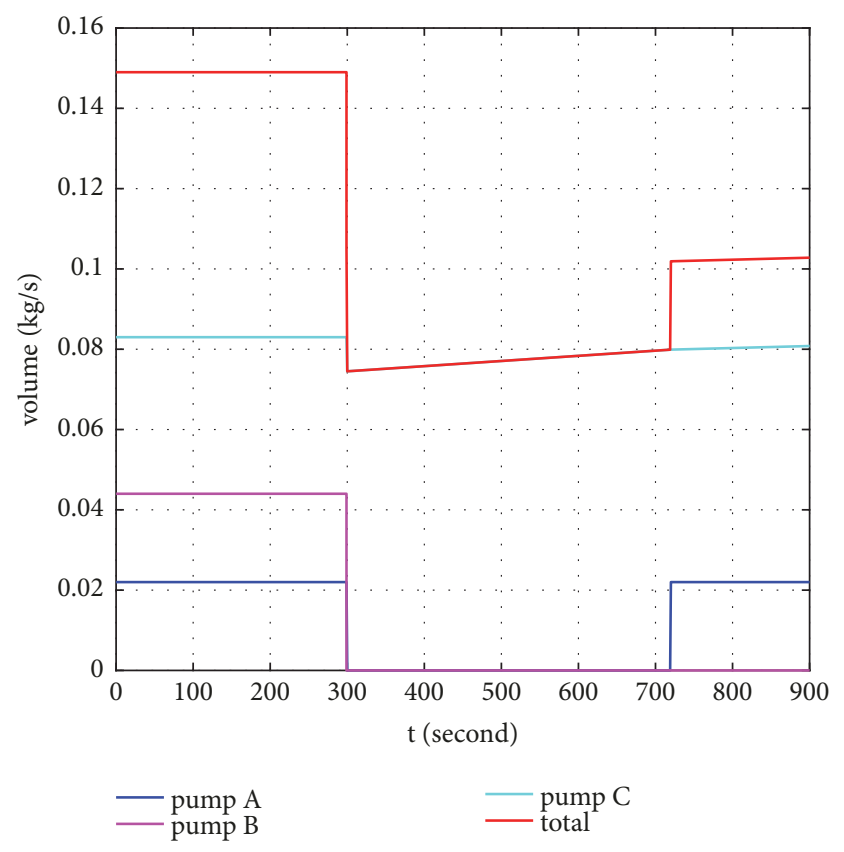

FIGURE 8: Flow volume under strategy 2.

minutes, the indoor temperature is always stable near the set point under the ideal strategies (the strategies 1 and 2), even if the indoor cooling load changes significantly; but under the strategy 3 , the indoor temperature expresses lower and higher instable rates in the last two time intervals, respectively.

\section{Conclusion}

In this paper, a type of linear multiswitched system with parallel structure was proposed, and the framework and a switching unit were introduced. Based on this, various actual engineering applications were shown, which illustrated the properties of the system and differences with traditional switched systems. Next, the stability property for a type of linear multiswitched system with parallel structure is studied, whether in continuous-time, discrete-time, or a mixed situation. A subsystems-group as a basic switched unit instead of subsystem is proposed, the matrices of which are pairwise commutative based on some given conditions of subsystems. When all the subsystems are Hurwitz and 
TABLE 4

\begin{tabular}{|c|c|c|}
\hline Parameter & Value & Description \\
\hline$m_{a}(\mathrm{~kg})$ & 232.18 & indoor air mass \\
\hline$q_{w}(\mathrm{~kg} / \mathrm{s})$ & 0.149 & rated volume of water system \\
\hline$q_{a}(\mathrm{~kg} / \mathrm{s})$ & 0.022 & rated volume of pump $A$ \\
\hline$q_{b}(\mathrm{~kg} / \mathrm{s})$ & 0.044 & rated volume of pump $B$ \\
\hline$q_{c}(\mathrm{~kg} / \mathrm{s})$ & 0.083 & rated volume of pump $C$ \\
\hline$q_{s a}(\mathrm{~kg} / \mathrm{s})$ & 3.003 & volume of sending air \\
\hline$A_{1}\left(\mathrm{~m}^{2}\right)$ & 56 & area of walls \\
\hline$A_{2}\left(\mathrm{~m}^{2}\right)$ & 28 & area of windows \\
\hline$A_{3}\left(\mathrm{~m}^{2}\right)$ & 0 & area of roof \\
\hline$C_{a}(\mathrm{~J} / \mathrm{kg} * \mathrm{k})$ & 1010 & specific heat of air \\
\hline$C_{w}(\mathrm{~J} / \mathrm{kg} * \mathrm{k})$ & 4180 & specific heat of water \\
\hline$K_{1}\left(\mathrm{~W} / \mathrm{m}^{2} * \mathrm{k}\right)$ & 0.049 & heat transfer coefficient of walls \\
\hline$K_{2}\left(\mathrm{~W} / \mathrm{m}^{2} * \mathrm{k}\right)$ & 0.051 & heat transfer coefficient of windows \\
\hline$K_{3}\left(\mathrm{~W} / \mathrm{m}^{2} * \mathrm{k}\right)$ & 0.05 & heat transfer coefficient of roof \\
\hline$Q_{q r}(J)$ & 20 & latent heat load \\
\hline$R_{r}$ & 0.11 & return air rate \\
\hline$\Delta \theta\left({ }^{\circ} \mathrm{C}\right)$ & 5 & temperature difference \\
\hline$\varepsilon$ & 0.89 & transfer efficiency from water system to air system \\
\hline$\gamma$ & 0.095 & coefficient of cooling capacity allocation \\
\hline$\theta_{\text {ini }}\left({ }^{\circ} \mathrm{C}\right)$ & $30,30,30$ & initial temperature \\
\hline$\theta_{j}\left({ }^{\circ} \mathrm{C}\right)$ & $35,35,36$ & temperature of walls, windows, and roof, respectively \\
\hline$\theta_{\text {set }}\left({ }^{\circ} \mathrm{C}\right)$ & 26 & setting temperature \\
\hline
\end{tabular}

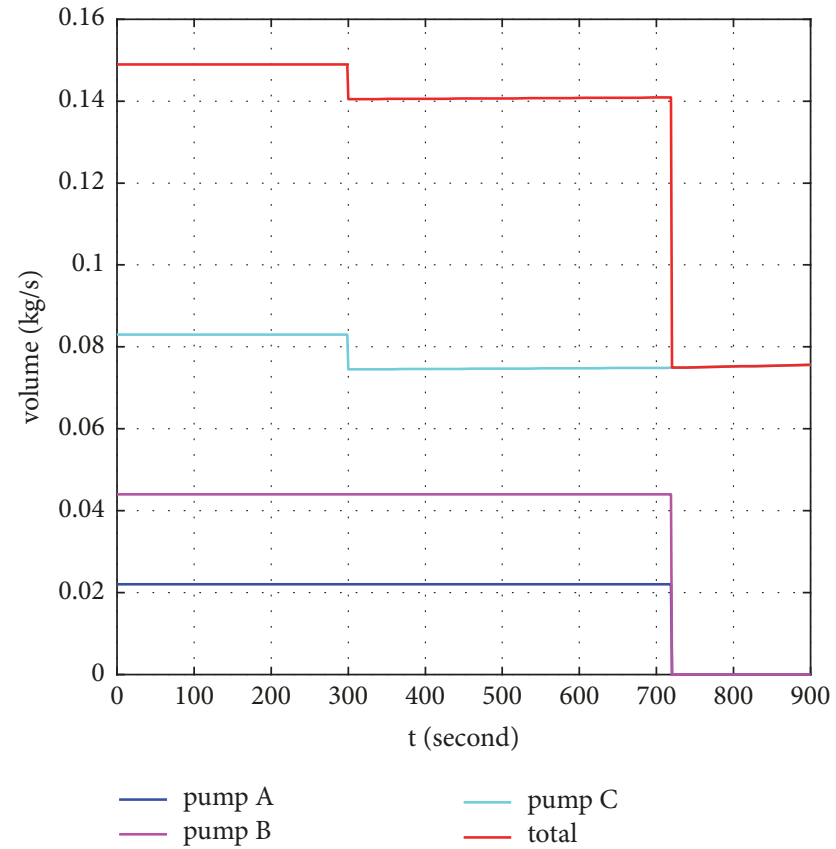

Figure 9: Flow volume under strategy 3.

Schur stable, there exists a common Lyapunov function for all the subsystems and subsystems-groups. Then, the switched system is exponentially stable for any arbitrary switching between the subsystems-groups. The results are extended to a parallel-like structure to obtain more ideal consequence of

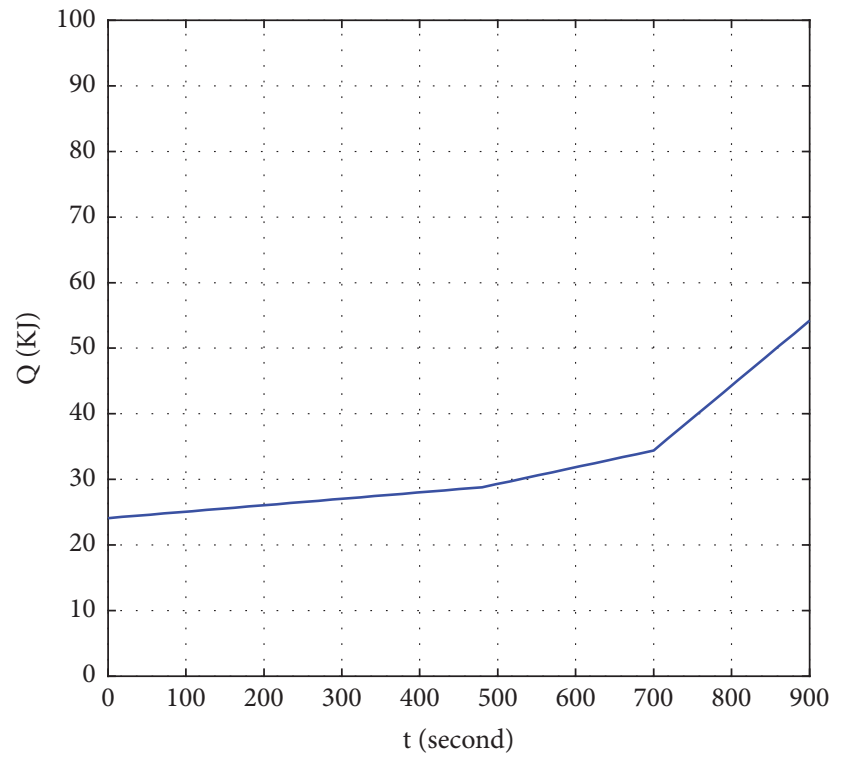

Figure 10: Change of cooling load.

stability. A simulation example for refrigeration engineering application of the system is introduced as last, which shows the characteristics of the framework and stability.

\section{Data Availability}

The data used to support the findings of this study are included within the article. 


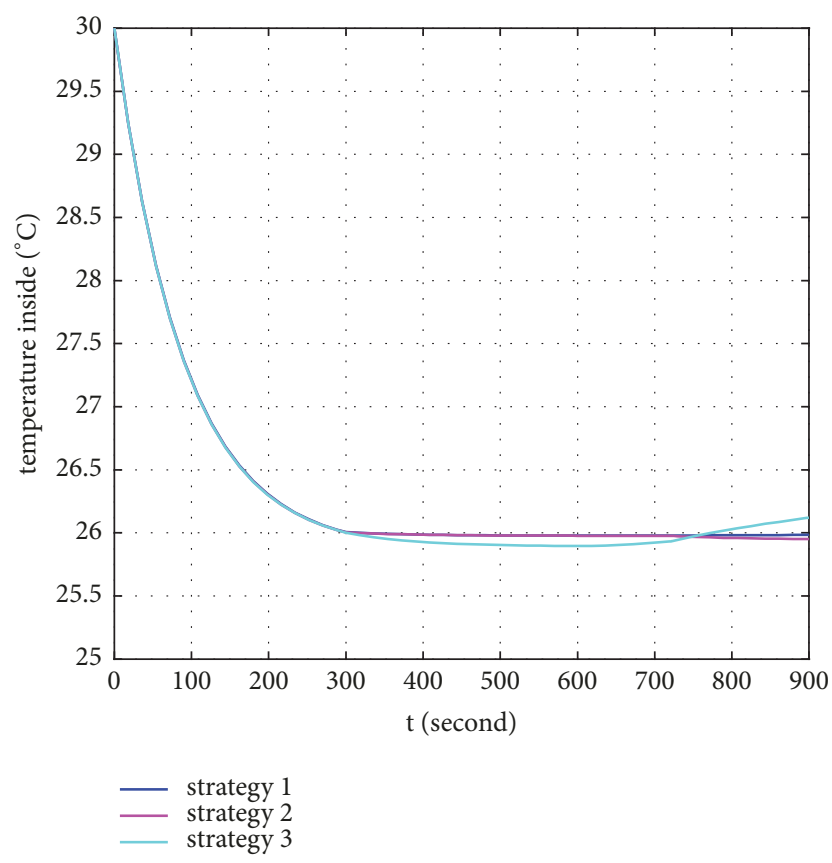

FIGURE 11: Indoor temperature under different strategies.

\section{Conflicts of Interest}

The authors declare that they have no conflicts of interest.

\section{Acknowledgments}

The research was supported by the National Natural Science Foundation of China (no. 61104181).

\section{References}

[1] G. Zhai, H. Lin, A. N. Michel, and K. Yasuda, "Stability analysis for switched systems with continuous-time and discrete-time subsystems," in Proceedings of the 2004 American Control Conference (AAC), pp. 4555-4560, July 2004.

[2] H. Lin and P. J. Antsaklis, "Stability and stabilizability of switched linear systems: a survey of recent results," IEEE Transactions on Automatic Control, vol. 54, no. 2, pp. 308-322, 2009.

[3] Z.-E. Lou and J. Zhao, "Stabilisation for a class of switched nonlinear systems and its application to aero-engines," IET Control Theory \& Applications, vol. 11, no. 2, pp. 237-244, 2017.

[4] Z. Sun and S. S. Ge, Stability Theory of Switched Dynamical Systems, Springer, London, UK, 2011.

[5] D. Liberzon, Switching in Cystems and Control, Birkhäuser, Boston, Mass, USA, 2003.

[6] R. Shorten, D. Leith, J. Foy, and R. Kilduff, "Towards an analysis and design framework for congestion control in communication networks," in Proceedings of the 12th Yale Workshop on Adaptive and Learning Systems, 2003.

[7] R. Shorten, F. Wirth, O. Mason, K. Wulff, and C. King, "Stability criteria for switched and hybrid systems," SIAM Review, vol. 49, no. 4, pp. 545-592, 2007.

[8] N. H. El-Farra and P. D. Christofides, "Coordinating feedback and switching for control of spatially distributed processes,"
Computers \& Chemical Engineering, vol. 28, no. 1-2, pp. 111-128, 2004.

[9] J. Jiang, K. Song, and Z. Li, "System Modeling and Switching Control Strategy of Wireless Power Transfer System," IEEE Journal of Emerging \& Selected Topics in Power Electronics, vol. 1-1, Article ID 99, 2018.

[10] L. Zhang, S. Zhuang, and R. D. Braatz, "Switched model predictive control of switched linear systems: feasibility, stability and robustness," Automatica, vol. 67, pp. 8-21, 2016.

[11] X. Liu, S. Li, and K. Zhang, "Optimal control of switching time in switched stochastic systems with multi-switching times and different costs," International Journal of Control, vol. 90, no. 8, pp. 1604-1611, 2017.

[12] J. Zhai, T. Niu, J. Ye, and E. Feng, "Optimal control of nonlinear switched system with mixed constraints and its parallel optimization algorithm," Nonlinear Analysis: Hybrid Systems, vol. 25, pp. 21-40, 2017.

[13] D. Liberzon and A. S. Morse, "Basic problems in stability and design of switched systems," IEEE Control Systems Magazine, vol. 19, no. 5, pp. 59-70, 1999.

[14] K. S. Narendra and J. A. Balakrishnan, "A common Lyapunov function for stable LTI systems with commuting A-matrices," IEEE Transactions on Automatic Control, vol. 39, no. 12, pp. 2469-2471, 1994.

[15] T. Büyükköroğlu, Ö. Esen, and V. Dzhafarov, "Common Lyapunov functions for some special classes of stable systems," Institute of Electrical and Electronics Engineers Transactions on Automatic Control, vol. 56, no. 8, pp. 1963-1967, 2011.

[16] R. A. Decarlo, M. S. Branicky, S. Pettersson, and B. Lennartson, "Perspectives and results on the stability and stabilizability of hybrid systems," Proceedings of the IEEE, vol. 88, no. 7, pp. 10691082, 2000.

[17] A. N. Michel, "Recent trends in the stability analysis of hybrid dynamical systems," IEEE Transactions on Circuits and Systems I: Fundamental Theory and Applications, vol. 46, no. 1, pp. 120134, 1999.

[18] L. Long and J. Zhao, "An integral-type multiple Lyapunov functions approach for switched nonlinear systems," Institute of Electrical and Electronics Engineers Transactions on Automatic Control, vol. 61, no. 7, pp. 1979-1986, 2016.

[19] J. P. Hespanha, "Chapter stabilization through hybrid control," Encyclopedia of Life Support Systems (EOLSS), 2004.

[20] D. Liberzon, J. P. Hespanha, and A. S. Morse, "Stability of switched systems: a Lie-algebraic condition," Systems \& Control Letters, vol. 37, no. 3, pp. 117-122, 1999.

[21] A. Sakly and M. Kermani, "Stability and stabilization studies for a class of switched nonlinear systems via vector norms approach,” ISA Transactions ${ }^{\circledR}, 2014$.

[22] G. Zhai and H. Lin, "Controller failure time analysis for symmetric $\mathscr{H}$ ocontrol systems," International Journal of Control, vol. 77, no. 6, pp. 598-605, 2004.

[23] G. Zhai, X. Xu, H. Lin, and A. . Michel, "Analysis and design of switched normal systems," Nonlinear Analysis. Theory, Methods \& Applications. An International Multidisciplinary Journal, vol. 65, no. 12, pp. 2248-2259, 2006.

[24] A. A. Agrachev and D. Liberzon, "Lie-algebraic stability criteria for switched systems," SIAM Journal on Control and Optimization, vol. 40, no. 1, pp. 253-269, 2001.

[25] J. L. Mancilla-Aguilar, "A condition for the stability of switched nonlinear systems," Institute of Electrical and Electronics Engineers Transactions on Automatic Control, vol. 45, no. 11, pp. 2077-2079, 2000. 
[26] R. N. Shorten and K. S. Narendra, "Necessary and sufficient conditions for the existence of a common quadratic Lyapunov function for M stable second order linear time-invariant systems," in Proceedings of the 2000 American Control Conference, pp. 359-363, June 2000.

[27] Yan Zhang, Yongqiang Liu, and Yang Liu, "A Hybrid Dynamical Modelling and Control Approach for Energy Saving of Central Air Conditioning," Mathematical Problems in Engineering, vol. 2018, Article ID 6389438, 12 pages, 2018. 


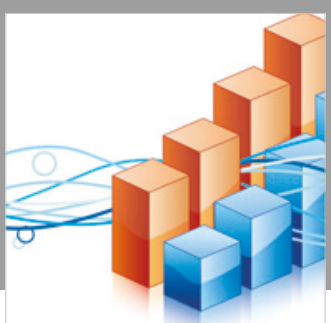

Advances in

Operations Research

\section{-n-m}
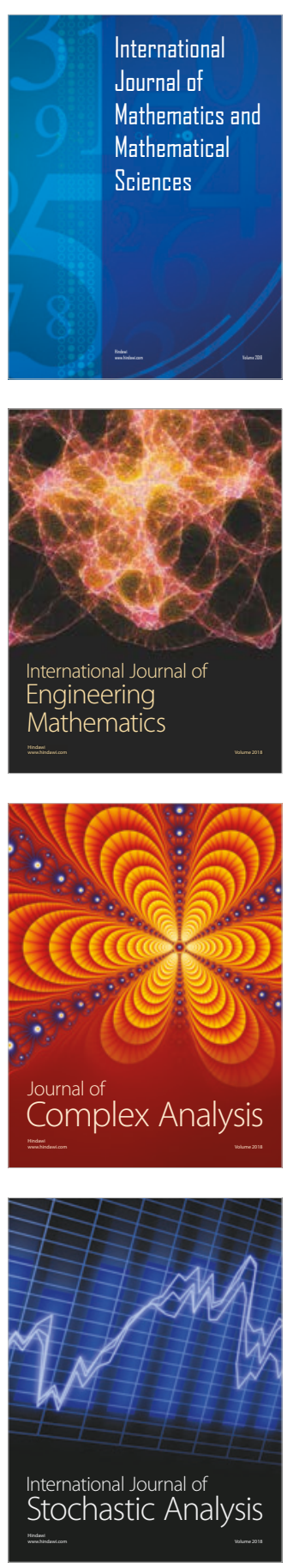
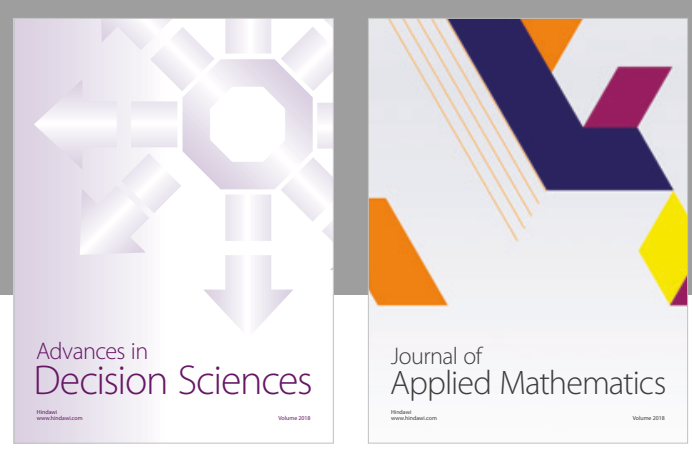

Journal of

Applied Mathematics
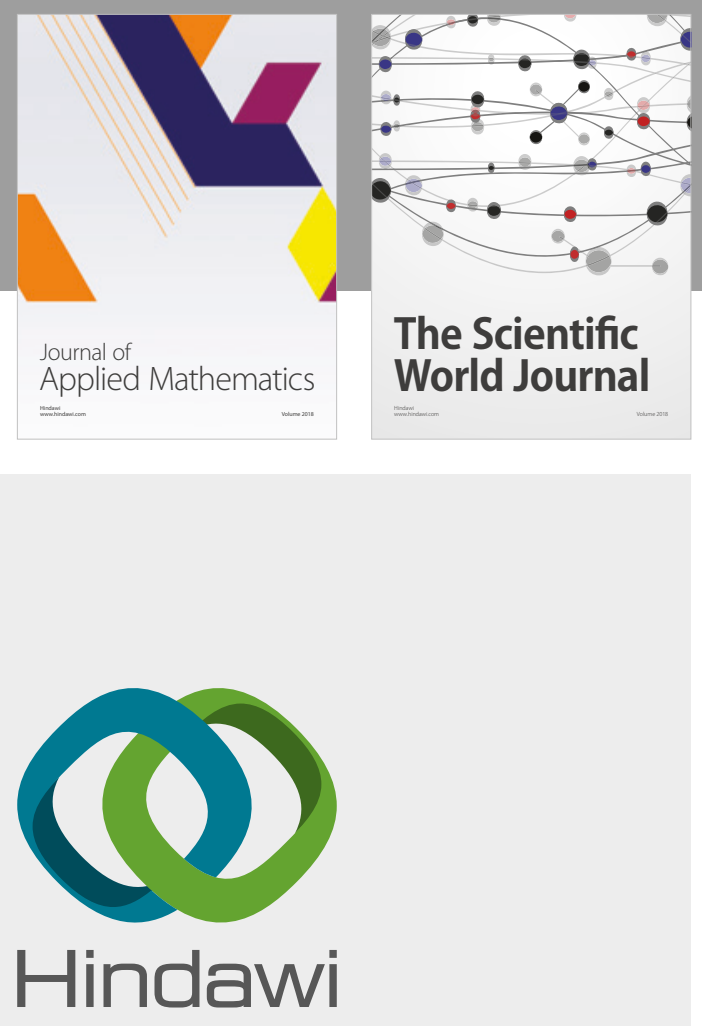

Submit your manuscripts at

www.hindawi.com

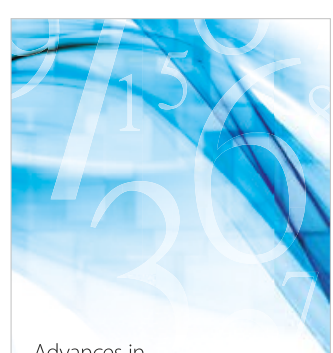

Advances in
Numerical Analysis
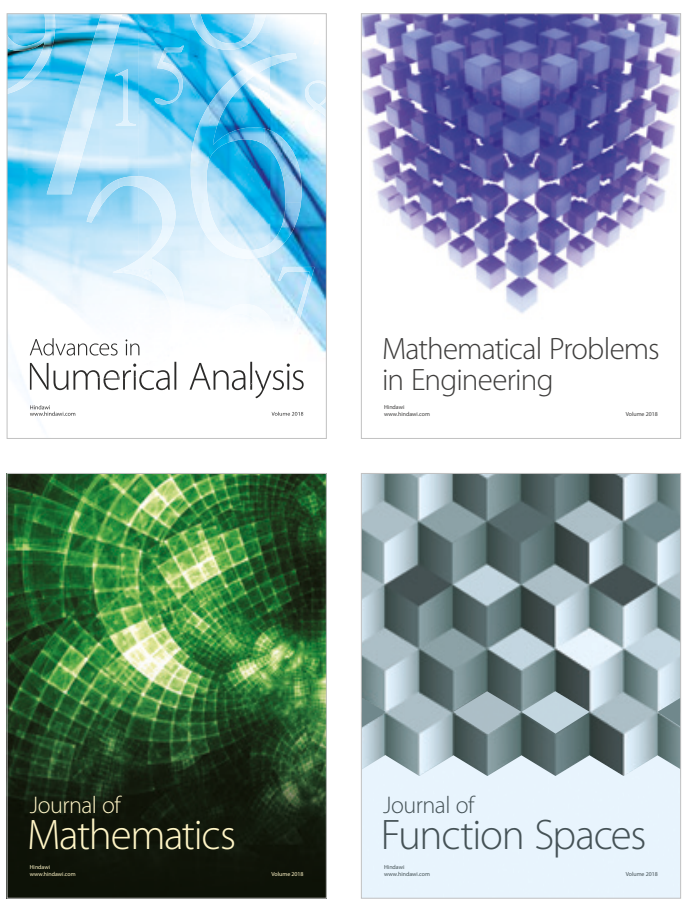

Mathematical Problems in Engineering

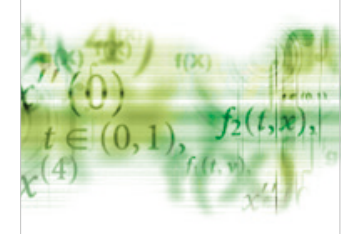

International Journal of

Differential Equations

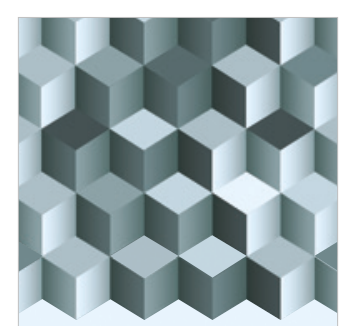

Journal of

Function Spaces

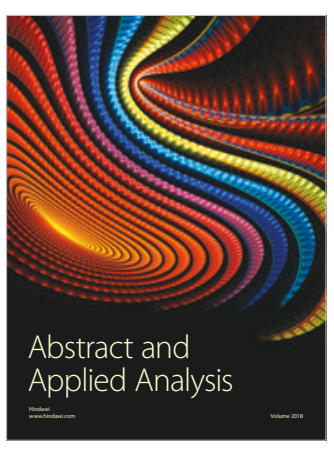

The Scientific

World Journal

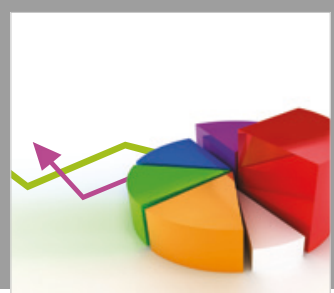

Journal of

Probability and Statistics
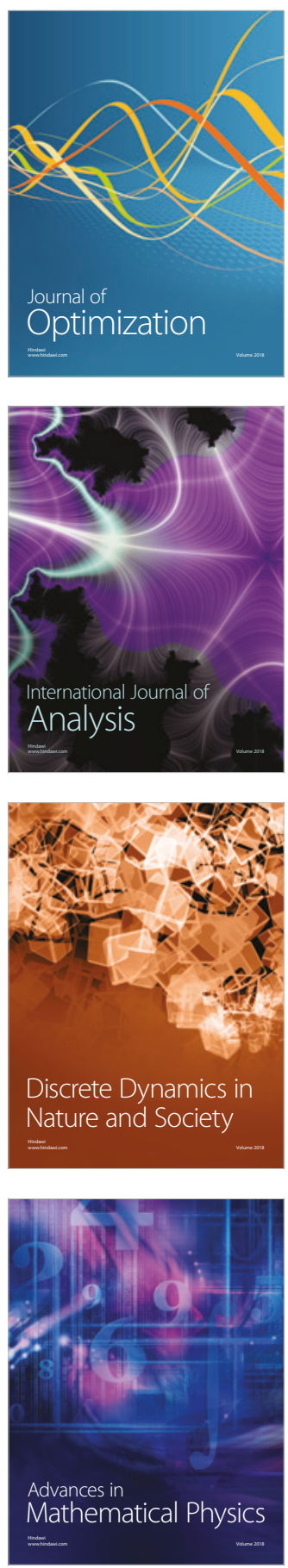